\title{
A Human-Robot Interaction Based Coordination Control Method for Assistive Walking Devices and an Assessment of Its Stability
}

\author{
Xia Zhang $\mathbb{D}^{1},{ }^{1}$ Wenliang Ge, ${ }^{1}$ Hao Fu, ${ }^{1}$ Renxiang Chen, ${ }^{1}$ \\ Tianhong Luo, ${ }^{1}$ and Minoru Hashimoto ${ }^{2}$ \\ ${ }^{1}$ Department of Mechatronics and Automobile Engineering, Chongqing Jiaotong University, No. 66 Xuefudadao, Nanan District, \\ Chongqing, China \\ ${ }^{2}$ Robotics Institutes, Shinshu University, 3-15-1 Tokida, Ueda, Nagano 386-8567, Japan \\ Correspondence should be addressed to Xia Zhang; zx512@126.com
}

Received 29 January 2018; Revised 7 May 2018; Accepted 5 July 2018; Published 18 July 2018

Academic Editor: Carlos Perez-Vidal

Copyright ( 2018 Xia Zhang et al. This is an open access article distributed under the Creative Commons Attribution License, which permits unrestricted use, distribution, and reproduction in any medium, provided the original work is properly cited.

\begin{abstract}
A biologically inspired motion control method is introduced to ameliorate the flexibility and multijoint autonomy of assistive walking devices based on human-robot interactions (HRIs). A new HRI-based coordination control system consisting of a hip central pattern generator (CPG) control, a knee hierarchical impedance control, and a hip-knee linkage control is also investigated. Simulations and walking experiments are carried out which demonstrate that (i) the self-oscillation and external communication characteristics of the CPG are capable of realizing ideal master/slave hip joint trajectories. In addition, symmetrical inhibition in the CPG unit is essential for maintaining the antiphase motion of the left and right hip joints. (ii) High and low hierarchical impedance control laws allow appropriate knee joint torque to be calculated to maintain posture during the support and swing phases as walking proceeds. (iii) A hip-knee joint linkage mechanism which incorporates a hip joint CPG control and knee joint impedance control allows natural and relevant hip-knee trajectories to be realized. The stability of the HRI-based coordination control method is also confirmed using Lyapunov stability theory.
\end{abstract}

\section{Introduction}

Since the 1960s, limb assistive devices such as orthoses and exoskeletons have undergone significant development. Such devices are designed with different architectures to assist their users' movements. Modern exoskeletons are essentially tools used to relieve the repetitive and heavy rehabilitation work imposed by physical therapists that improve the efficacy of the patient's recovery.

The Lokomat [1] and LOPES [2] exoskeletons are aimed at helping paraplegic or quadriplegic people to regain locomotive ability in their daily life. ATLAS [3], ReWalk [4], and Ekso [5] are augmentation systems adopted to empower otherwise healthy people to perform the carrying of heavy loads. The BLEEX [6], Sarcos [7], and MIT [8] exoskeletons are mainly used to provide additional power to enable people suffering from muscular weakness (e.g., the elderly) to walk or climb stairs. Hybrid assistive limb (HAL) [9] and Honda's Stride Management Assist system (Honda, Tokyo, and Japan) are also helping people walk again. The latter emerging field of application is a consequence of population aging in industrialized countries [10] and low birth rates and high-life expectancy in these countries [11]. The aging of populations has resulted in considerable attention being focused on how to provide flexible master/slave assistance for the elderly in their daily lives, depending on their physical condition.

Assistive strategies can be divided into six categories: sensitivity amplification, predefined gait trajectory control, model-based control, oscillator-based control, predefined action based on gait pattern, and hybrid assistive strategies. The first, sensitivity amplification, is mostly used to handle exoskeletons which increase the load-carrying capability of a user. In this strategy, the controller usually relies on an inverse dynamic model of the exoskeleton. The force exerted by the 
wearer on the exoskeleton is set on a positive feedback loop of the controller and can be scaled down according to an amplification parameter $[12,13]$.

With predefined gait trajectory control mechanisms, the desired joint trajectory is either prerecorded using a healthy person or extrapolated from a gait analysis data atlas and is then replayed on the user's exoskeleton $[14,15]$. Under a model-based control structure, the desired robotic action is computed using a human-exoskeleton model. The strategy usually aims to include gravity compensation and zero moment point balance criteria and provide extra commanded assistance [16, 17]. Although straightforward, this control strategy relies on the accuracy of the model which is used and requires a series of sensors to recognize kinematic and dynamic variables.

Adaptive oscillators are mathematical models that are a particular kind of central pattern generator (CPG). A learning mechanism for them was first developed by Righetti et al. in order to synchronize their frequency with the instantaneous frequency and phase of any periodic input signal [18]. These models have been widely used in the robotics field $[19,20]$. Hybrid assistive strategies aim to control the exoskeleton by applying different assistive strategies.

Assistive and rehabilitation robotics will naturally have different objectives and use different assistive strategies. However, the human-robot interaction (HRI) is a critical issue in both applications. Indeed, the HRI is responsible for both power and information transmission.

In general, a single control method cannot be the best option to use for all the different motions encountered. Instead, a comprehensive range of control strategies is required for the assistive controller to provide natural and appropriate assistance to humans. Strategies such as modelbased control and predefined action based on gait pattern are considered adequate in cases where arbitrary motion is involved. High impedance control of predefined gait trajectory methods can be adequate in cases where the motion to be made is relatively small and the force necessary is large. The daily motion of humans, such as walking, sitting, and standing, consists of combinations of these different cases. Therefore, the controller of the assistive device is required to be capable of conducting a comprehensive range of strategies as well as switching between them according to the motion desired.

Hybrid control schemes have been applied to various assistive robots in recent years. For example, HAL (a fullbody assistive robot developed with multiple purposes) uses a hybrid control that changes the control algorithm according to the phase of the motion as walking proceeds. In this case, the phase of the motion is detected according to the pattern of the ground reaction force and classified by the type of muscular activity involved [21]. The BLEEX exoskeleton also uses a hybrid control method to assist the walking motion. The controller of the BLEEX system has two main subcontrol algorithms, one in which a master-slave control method is activated (for stance phases), and one in which a sensitivity amplification control method is used (for swing phases). Foot-pressure patterns are also used in the BLEEX system to detect the phase of the motion [22].
Oh et al. (2015) proposed a generalized control framework incorporating various assistive control methods. The framework consists of a feedforward disturbance compensation control, reference tracking feedback control, reference tracking feedforward control, and a model-based torque control. The proposed control framework is designed taking into consideration the linearity of each control algorithm to enable continuous and smooth switching between assistive control algorithms [23]. Vijaykumar developed a control strategy that provides the necessary assistance required and is related to balance recovery and postural stability [24].

The aforementioned hybrid control methods essentially consist of traditional impedance control, compliance control, and relative stability strategies. Even though these hybrid methods have been successfully implemented in various assistive robots, and their performance has improved with practice, none of the researches has been able to avoid kinematic and dynamic modulation. As a consequence, problems have emerged related to the complicity and stability of the systems when switching strategies. In order to address this problem, and to improve the flexibility and multijoint autonomy in walking assist applications, this paper attempts to provide a coordination control framework for the HRI. The proposed framework incorporates HRI technology based on CPG networks and traditional impedance structured control.

In previous studies, the validity and feasibility of the application of CPGs to assistive devices have been evaluated experimentally. The experimental results have confirmed that CPG-based control enables master/slave motion to be achieved and provides an assist effect as well [25]. Nevertheless, in walking assistance applications, two problems-how to provide stable assistance and how to achieve multijoint autonomy during walking-remain important issues. In this paper, a CPG network is used to facilitate synchronization between the assistive robot and human, as well as generating the desired trajectory for the hip joint. In order to keep an antiphase relationship between the left and right hip joints, mutual inhibition between the left and right oscillators is incorporated into the control system. A high and low hierarchical impedance control law is also designed for control of the knee joint. As a result, the knee joint torques required during the support and swing stages are obtained according to the gait requirements. Finally, a hip-knee joint linkage mechanism is built that incorporates the hip joint CPG control and knee joint impedance control. Thus, natural and relevant hip-knee joint trajectories are realized. The human-robot interaction based coordination control framework was firstly proposed in our previous study [26], and the rationality of the proposal was examined preliminarily using simulation analysis. In this paper, we conduct a series of further simulations and walking experiments using a robotic suit with four degrees of freedom to verify the validity of our proposed method and application (a wearable robot to achieve natural hip and knee joint motion during walking).

The paper is structured as follows. In Section 2, we introduce the framework for the HRI-based coordination control method which consists of a hip central pattern generator (CPG) control, a knee hierarchical impedance control, and a hip-knee linkage control. We analyze the stability of the 


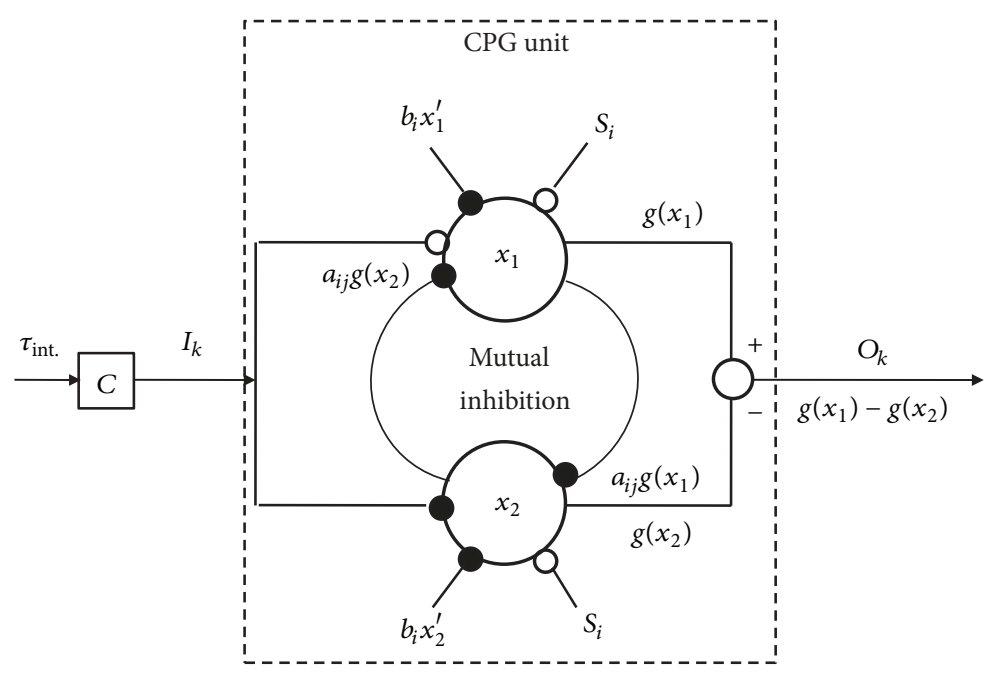

FIgUre 1: The structure of a single CPG unit.

proposed control system. This section also presents computer simulations to investigate the stability of the control system. In Section 3, we conduct walking experiments and discuss the dynamic performance. The discussion is provided in Section 4. Finally, conclusions of this study and outline of future work are given in Section 5.

\section{HRI-Based Coordination Control}

This section provides details of the HRI-based coordination controller, which is composed of three parts. Firstly, there is a CPG-based hip joint control that serves to achieve synchronization between the assistive robot and human. The CPG returns the robot's desired hip joint angle (master/slave motion mode, depending on the users' physical condition). Secondly, there is a knee joint hierarchical impedance control which serves to calculate the knee joint torque required for the stance and swing stages. Finally, a knee joint and hip-knee joint linkage mechanism is implemented in order to achieve the required motion of the hip-knee joint.

\subsection{CPG-Based Hip Joint Control}

2.1.1. CPG Model. In this work, CPGs are used to generate joint trajectories. Following the work of Matsuoka [27], a CPG unit (an oscillator) consisting of two neurons is designed: one is an extension neuron, and the other is a flexion neuron. The two neurons correspond to the extensor and flexor muscles in animals, respectively (Figure 1). A mathematical model can be constructed for the unit which can be expressed via the coupled equations

$$
\begin{aligned}
& T_{r} \frac{\mathrm{d} x_{i}}{\mathrm{~d} t}+x_{i}=-\sum_{j \neq i} a_{i j} g\left(x_{j}\right)+S_{i}-b_{i} x_{i}^{\prime}+(-1)^{i-1} I_{k} \\
& T_{a} \frac{\mathrm{d} x_{i}^{\prime}}{\mathrm{d} t}+x_{i}^{\prime}=g\left(x_{i}\right), \quad g\left(x_{i}\right)=\max \left(0, x_{i}\right)
\end{aligned}
$$

For the sake of clarity, the notation and definitions used in (1) and (2) are presented in Table 1.
TABLE 1: The definitions of the parameters appearing in (1) and (2).

\begin{tabular}{lc}
\hline Parameter & Definition \\
\hline$x_{i}$ & The inner state of the $i$-th neuron $(i=1$ or 2$)$ \\
$x_{i}^{\prime}$ & $\begin{array}{c}\text { Self-suppression parameter for the } i \text {-th neuron } \\
\text { Time constant for the inner state of the } i \text {-th } \\
\text { neuron }\end{array}$ \\
$T_{\mathrm{r}}$ & Time constant for the adaptation of the $i$-th \\
neuron
\end{tabular}

In Figure 1, the output of the CPG unit is calculated using the formula $g\left(x_{1}\right)-g\left(x_{2}\right)$, or equivalently $\max \left(0, x_{1}\right)-$ $\max \left(0, x_{2}\right)$, to obtain a series of continuously oscillating signals. The output of the CPG corresponds to the desired trajectory of the hip joint of the assistive robot in this article.

The Matsuoka model is nonlinear with complex characteristics (strong coupling and high dimension). As a result, it is not possible to give an exact theoretical solution to the problem, nor is it possible to give a full description of the effects of the parameters on the unit's output. In engineering applications, therefore, numerical computer simulations are widely used to find the rules governing how a specific parameter affects the output. According to the rules thus obtained, parameters can then be assigned relevantly and directly. Therefore, in this article, typical CPG parameters are determined based on the premise of combining stable oscillation with the gait requirements needed (more specifically, with reference to the frequency of the hip joint motion associated with healthy walking adults). 
The values of the coefficients are subsequently assigned to be $a_{12}=a_{21}=1.2, b_{i}=2.5, s_{i}=2.0, T_{\mathrm{r}}=0.12$, and $T_{\mathrm{a}}=$ 0.6. As long as the parameters mentioned above are defined, the two neurons will be activated alternately at a certain frequency, which is about $1.0 \mathrm{~Hz}$. The input to the oscillator is a kind of measurement signal (interaction torque in this article) multiplied by the input weight $C$. If there is no input to be fed back, the oscillator will output a stable sinusoidal signal as a result of the aforementioned parameter assignments. Once any input is fed back, the neural oscillator will export output signals that are synchronized with the input signals.

The input weight coefficient $C$ is introduced to adjust the coupling strength between the torque of the human-machine interaction and the CPG's self-excited oscillation. The input function of the CPG unit can be expressed using the following equation:

$$
I_{k}=C \cdot \tau_{\text {int }}
$$

where $\tau_{\text {int. }}$ is the interaction torque fed back to the $k$-th CPG unit (which is generated as soon as there is any difference between the user's and the suit's movement) and $I_{k}$ is the amplitude of input signal. Basically, $I_{k}$ should be in the interval $[-1,1]$ to obtain stable and continuous master/slave movement.

Therefore, the value of $C$ should be assigned to guarantee $I_{k}$ to be on the interval $[-1,1]$ according to the magnitude of the interaction torque. By changing $C$, the amplitude of the input to each CPG will be increased or decreased. Consequently, different outputs will be obtained. Increasing the gain will enable the CPG to be entrained and thus synchronize with the input signal. Conversely, decreasing the gain will weaken the input signal so that the CPG will tend to lose synchronization and undergo autonomous oscillation. In other words, the value of the gain $C$ determines whether the robot's joint motion corresponds to master or slave. Furthermore, the larger the value of $C$ is, the more easily an assistive device moves synchronously with the human motion. (Conversely, the smaller its value is, the more difficult it is for the assistive device to move synchronously with the human motion-rather it will become the master and move in autonomous oscillation mode.)

Figure 2 shows the output behavior of a CPG for different values of $C(0.1,0.3$, and 0.8$)$. The plots on the left correspond to the input to output transformation results; those on the right represent the limit cycles of the output oscillation. The dashed lines in the I/O graphs represent the input to the CPG, which takes the form of the sinusoidal function $\sin (1.4 \pi t)$. The solid line represents the output of the CPG. Comparing the results of the simulations, Figure 2(a) shows that the self-oscillation of the CPG is maintained and the oscillation frequency is about $1.0 \mathrm{~Hz}$ (this corresponds to the low $C$ result). Figure 2(c), on the other hand, shows that in this case (high $C$ value) the output frequency of the CPG is changed from the original oscillation frequency of $1.0 \mathrm{~Hz}$ to $0.7 \mathrm{~Hz}$. In addition, the amplitude is also increased. Figure 2(b) corresponds to an "intermediate" transition state somewhere between self-oscillation and synchronous oscillation (due to the "intermediate" value of $C$ used).
The limit cycles shown in (a) and (b) show that the CPG unit can easily transform from a stable autonomous oscillation mode to another stable oscillation mode that is synchronous. The above results thus reveal that the proposed method is applicable to master-slave motion.

The output of the CPG unit corresponds to the desired angle $\theta_{\mathrm{d}}$ of the robot joint which can be calculated using the expression

$$
\theta_{\mathrm{d}}=\theta_{0}+K_{\mathrm{a}} \cdot \mathrm{O}_{k}
$$

where $O_{\mathrm{k}}$ is the output from the CPG unit (in Nm), $K_{\mathrm{a}}=$ $28^{\circ} /(\mathrm{Nm})$ is a constant used to convert between torque and angle and to tune the amplitude, and $\theta_{0}=15^{\circ}$ is an offset used to emulate the natural motion of the hip joint. The values of $K_{\mathrm{a}}$ and $\theta_{0}$, of course, need to be determined according to the requirements of the system involved. In this article, the values of $K_{\mathrm{a}}$ and $\theta_{0}$ are, in the early stages, properly assigned to correspond to natural hip joint trajectories according to experimentally determined walking data [20].

2.1.2. Mutual Inhibition between Two CPGs. To walk stably, the joints in the legs of humans have to maintain a certain difference in phase. Zhang et al. (2011) revealed, in fact, that the left and right hip joints have to have an antiphase relationship. It is therefore necessary for robotic suits to obey this relationship as well in order to confer stability to the walking assistance provided. That is, there should be a kind of human-like cooperative motion between each joint in the robotic suit. To address this requirement, we propose incorporating mutual inhibition between the CPG units.

Mutual inhibition between the neurons will cause them to activate alternately. Figure 3 depicts a pair of oscillators between which mutual inhibition can be achieved. In this case, the number of neurons is four, and the number of oscillators is two (as before, $a_{12}=a_{34}=1.2$ ). The synchronization gain $C$ controls the outer synchronization, and the newly introduced inhibitory weights $a_{13}=a_{24}=0.12$ control the inner-inhibition. The choice of identical inhibitory weights $\left(a_{13} \equiv a_{24}\right.$ ) has been discussed previously by Zhang et al. (2012). This outer-synchronization/inner-inhibition mechanism is repeated throughout the assisted walking process to stabilize the assisted motion.

The output from the pair of CPGs, with and without mutual inhibition, is compared in Figure 4. In the figure, two different input signals are used: input 1 (represented by the blue solid line in the upper part of the figure) which is a normal sinusoidal curve and input 2 (represented by the dotted line) which is partly sinusoidal and partly constant with a zero value). The inputs are then set to zero from $5 \mathrm{~s}$ onwards. The light region in Figure 4 indicates $C=0$ and the dark region $C=1$.

In the case without mutual inhibition, the CPGs individually synchronize with the different input signals (the outputs shown by the black solid line in Figure 4). However, when the different input signals become zero at $5 \mathrm{~s}$, the original antiphase relationships become disordered and the original antiphase relationship is not retained.

In the case with mutual inhibition, the CPGs synchronize with the input signals as well. What is important in this case 

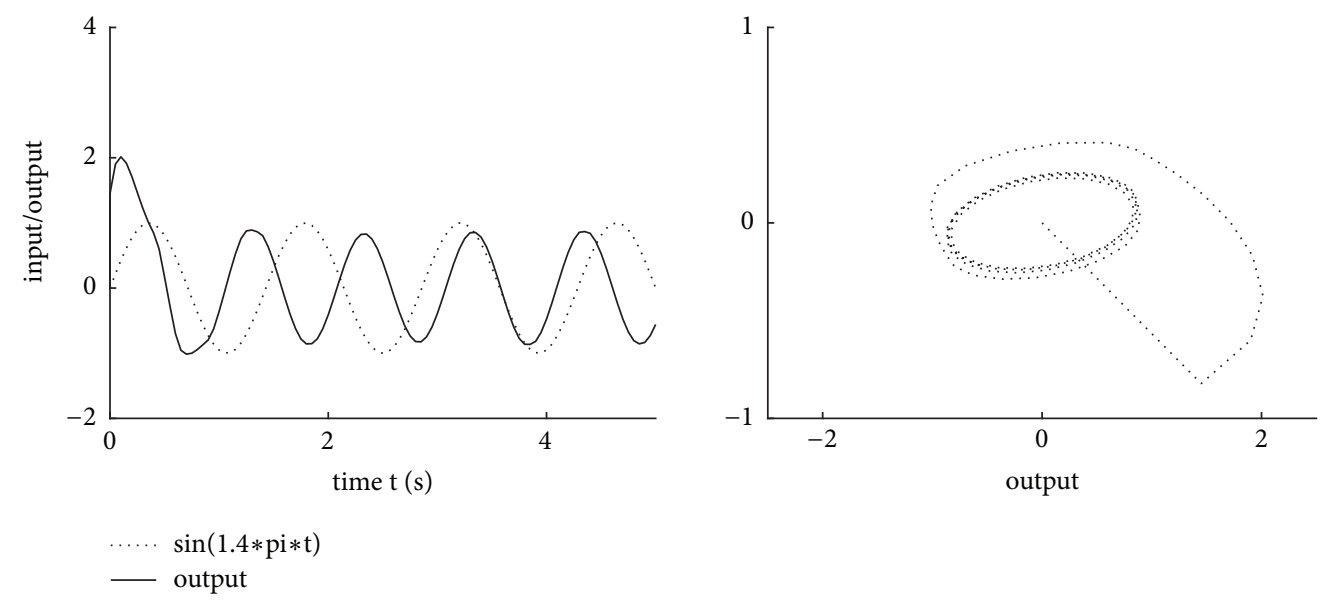

(a)
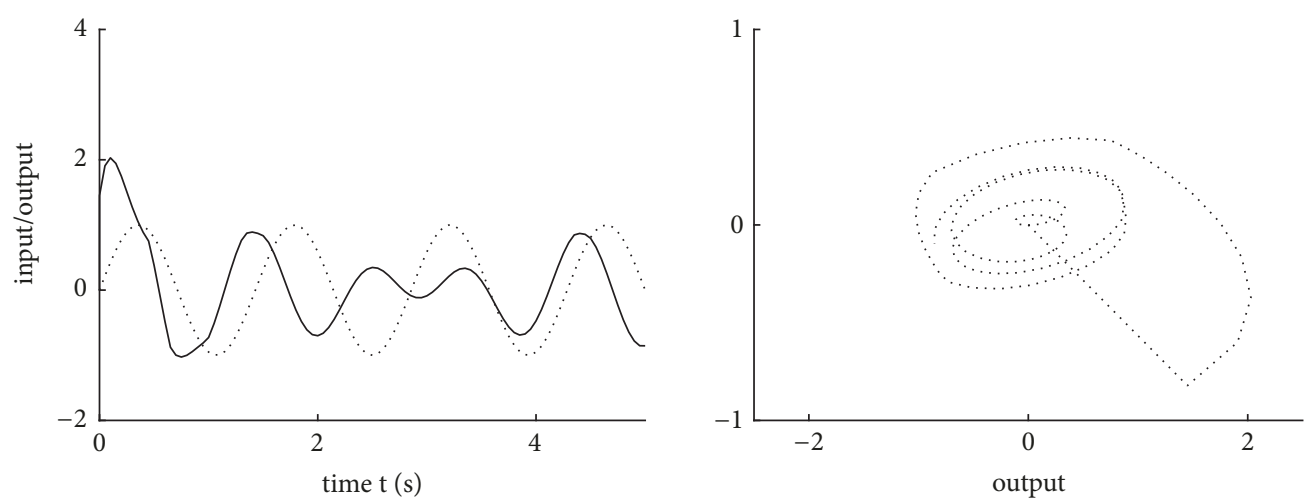

$\begin{array}{ll}\cdots & \sin (1.4 * \mathrm{pi} * \mathrm{t}) \\ & \text { output }\end{array}$

(b)
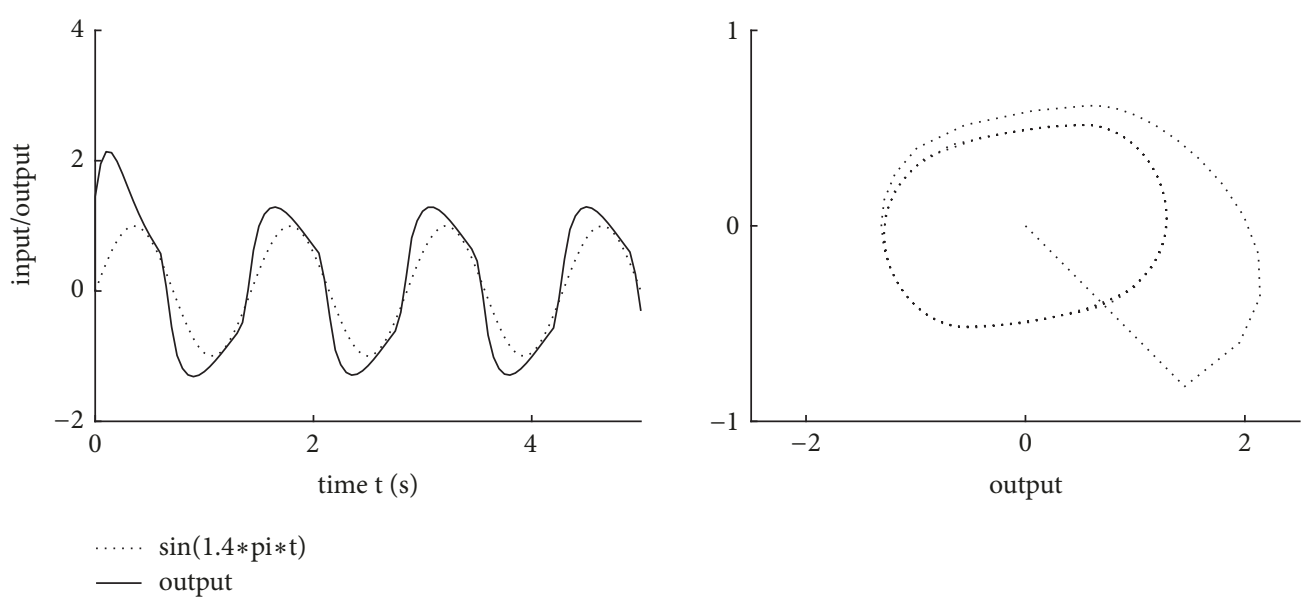

(c)

FIgURE 2: The input-output characteristics of the CPG unit (left) and output limit cycles (right) for different gain values corresponding to C equal to (a) 0.1, (b) 0.2 , and (c) 0.8 .

is that the behavior of the CPG units (blue dotted line in Figure 4) returns to the original antiphase relationship immediately and the input signals disappear. The autonomous, antiphase behavior of the robotic suit thus helps the user to walk in a stable manner.
2.1.3. CPG-Based Hip Joint Control. In the previous section, the CPG network's external communication mechanism converts the interaction torque to the desired master/slave trajectory according to the value of $C$. It is also responsible for the communication between the assistive device and 


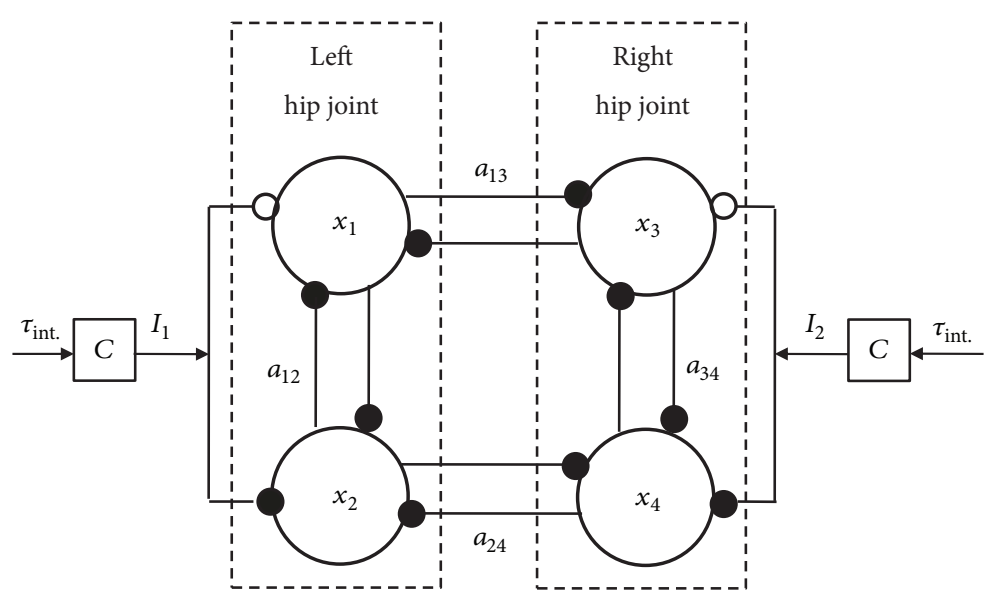

Figure 3: A CPG network with mutual inhibitory functionality.
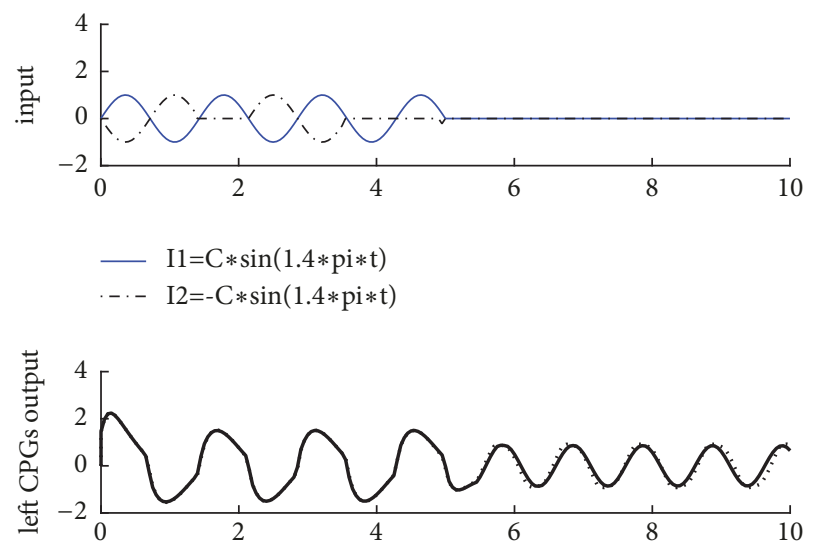

— without inhibition ..... with inhibition

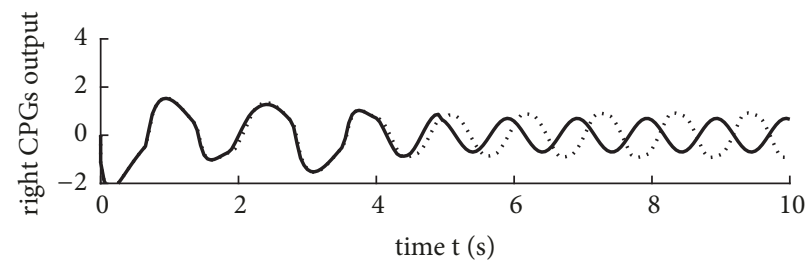

FIgURE 4: Antiphase autonomic regression of the left and right CPG units.

human being at the hip joints. Meanwhile, the internal inhibition mechanism of the CPG network guarantees the output oscillations of the left and right CPG units are antiphase with respect to each other (so the left and right hip joints move with the desired antiphase motion). A proportional derivative (PD) controller is used to realize control of the joint trajectory, and the motion differences between the human and machine generate new interaction torques. A block diagram for the control system is shown in Figure 5.
In each control cycle, three aspects are considered: human movement intension, human-robot interaction, and multijoint autonomy. As the output signal of the CPG is synchronized with the frequency of the input signal, when the oscillation frequency of the assistive device is close to the oscillation frequency of the human, resonance conditions are encountered. The traction force between the human and machine will therefore amplify the amplitude of the system oscillation to achieve motion assistance that is highly efficient. The torque $\tau_{\text {mea. }}$ in Figure 5 is measured using a built-in torque 


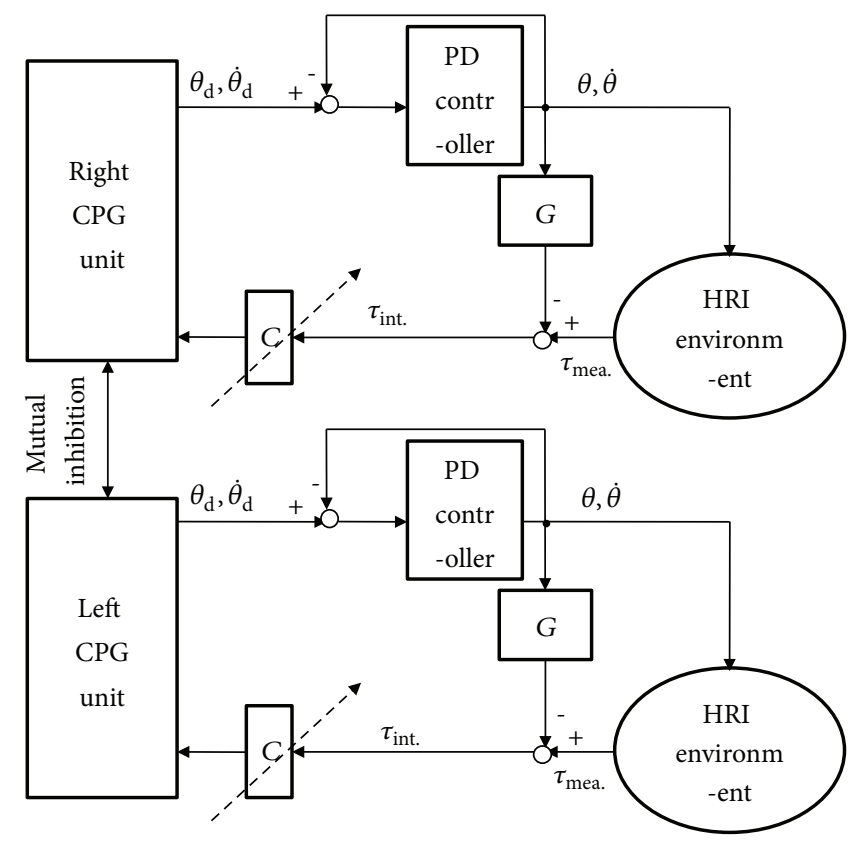

FIGURE 5: The framework used to form the CPG-based hip joint control system.

sensor, and $G$ is the gravity compensation unit. The humanrobot interaction torque $\tau_{\text {int. }}$ is calculated using the formula:

$$
\tau_{\text {int. }}=\tau_{\text {mea. }}-G
$$

The PD position control algorithm can be represented mathematically using the expression:

$$
\tau=K_{\mathrm{p}}\left(\theta_{\mathrm{d}}-\theta\right)+K_{\mathrm{d}}\left(\dot{\theta}_{\mathrm{d}}-\dot{\theta}\right)
$$

where $K_{\mathrm{p}}=20.0 \mathrm{Nm} /\left(^{\circ}\right)$ and $K_{\mathrm{d}}=1.5 \mathrm{Nm} \cdot \mathrm{s} /\left(^{\circ}\right), \theta_{\mathrm{d}}$ and $\theta$ represent the desired and actual joint angles of the assistive device, respectively, and $\dot{\theta}_{\mathrm{d}}$ and $\dot{\theta}$ represent the corresponding angular velocities, respectively. The sampling time used is $1 \mathrm{~ms}$.

2.2. Knee Joint Hierarchical Impedance Control. In contrast to hip joints, the motion of knee joints is much more complicated. The requirements are as follows. Firstly, the knee joint keeps straight to support the whole body weight (stance phase), and then it flexes and extends (swing phase). Secondly, knee joints require large stiffness (high impedance) in the stance phase but are compliant (low impedance) in the swing phase. According to the aforementioned gait requirements, a framework for high and low impedance hierarchical control laws can be designed for the control of knee joint motion in the stance and swing phases, respectively.

The high and low impedance control laws can be expressed mathematically in the form

$$
\begin{aligned}
& \tau_{\text {kneehigh }}=-K_{\mathrm{p}}\left(\theta_{\mathrm{k}}-0\right)-K_{\mathrm{d}} \dot{\theta}_{\mathrm{k}} \\
& \tau_{\text {kneelow }}=K_{\text {amp }} \tau_{\text {int. }}
\end{aligned}
$$

where $K_{\mathrm{p}}$ and $K_{\mathrm{d}}$ are PD controller gains, $\theta_{\mathrm{k}}$ and $\dot{\theta}_{\mathrm{k}}$ are the knee joint angle and velocity, respectively, and $K_{\mathrm{amp}}$ is an amplifier gain. The control law represented by (8) allows the knee joint to be compliant with the human-robot interaction force. These equations allow the required knee joint torques $\tau_{\text {kneehigh }}$ and $\tau_{\text {kneelow }}$ to be calculated for the stance and swing phases, respectively, as walking proceeds.

2.3. Hip-Knee Joint Linkage Mechanism. While walking, a human being's hip and knee joints undergo distinctive and interdependent motion. For example, while the hip joint is in a state of extension in the stance phase, the knee joint of the ipsilateral leg is kept straight to support the whole body weight. In the swing phase, the hip joint is flexing, while the knee joint first undergoes flexion and then extension. In order to realize this natural hip-knee joint interdependent motion, a hip-knee joint linkage mechanism is designed, as shown in Figure 6.

As the extension and flexion neurons of a given oscillator become active alternately, the states of the neurons can be used as variables to generate activation functions. Two activation functions are employed defined by the following equations:

$$
\begin{gathered}
g_{\text {ext }}\left(x_{i}\right)=\max \left(x_{i}, 0\right), \quad i=1 \text { or } 3, \\
g_{\text {flex }}\left(x_{j}\right)=\max \left(x_{j}, 0\right), \quad i=2 \text { or } 4 .
\end{gathered}
$$

The function $g_{\text {ext }}\left(x_{i}\right)(i=1,3)$ thus associates the hip joint extension motion with the knee joint high impedance law, and $g_{\text {flex }}\left(x_{j}\right)(j=2,4)$ associates the hip joint flexion motion with the knee joint low impedance law. This kind of linkage is expected to allow the knee joint to execute high and low impedance control laws along with the hip joint's extension and flexion motion. Thus, a series of natural hip-knee joint interdependent movements can be accomplished. 


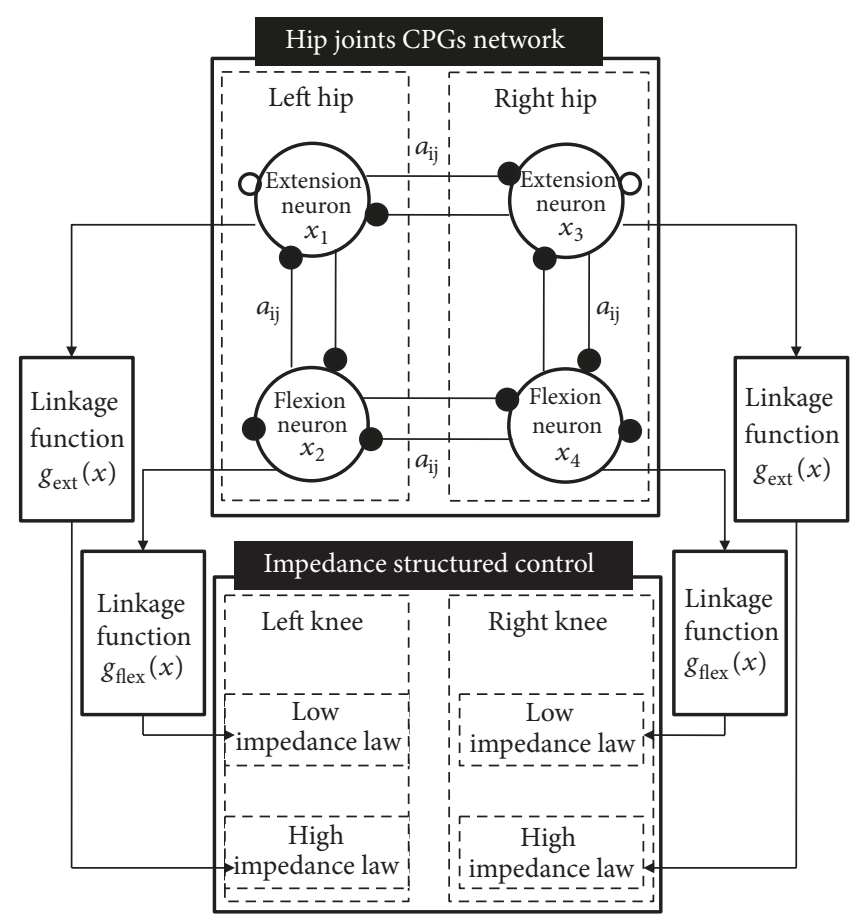

Figure 6: The hip-knee joint linkage mechanism.

The hip-knee joint linkage mechanism is represented using the following expression:

$$
\begin{aligned}
\tau_{\text {knee }}= & g_{\text {ext }}\left(x_{i}\right) \cdot\left[-K_{\mathrm{p}}\left(\theta_{\mathrm{k}}-0\right)\right]-K_{\mathrm{d}} \dot{\theta}_{\mathrm{k}}+g_{\text {flex }}\left(x_{j}\right) \\
& \cdot \tau_{\text {kneelow }}
\end{aligned}
$$

where $i=1,3$ and $j=2,4$. The high and low impedance control laws are activated and adjusted during the stance and swing phases by the extending $g_{\text {ext }}\left(x_{i}\right)$ and bending $g_{\text {flex }}\left(x_{i}\right)$ activation functions, respectively.

The continuous differentiability of the neuron state benefits the high and low impedance control laws by maintaining continuity during switching. In short, the alternate excitation mechanism of the extension/flexion neurons and continuously differentiable characteristics enable the hipknee linkage control to activate or inhibit the high and low impedance control laws smoothly and continually. As a result, the knee joint can keep upright as the hip joint extends and the lower thigh can move forward naturally as the hip joint bends. Therefore, the smooth and continuous switching of the control laws ensures the stability of the control system.

2.4. Stability Study. The proposed HRI-based coordination control method is a hybrid control system composed of several control methods. Therefore, the stability of the control system must be confirmed first. As traditional PD controllers are used in the hip joint for trajectory-tracking control, the key to the stability of the whole control system is the stability of the switching process between the high and low impedance control of the knee joint. In this paper, Lyapunov stability theory is used to study and evaluate the stability of the switching process and thus to discuss the stability of the proposed coordination control method. We take the left knee joint as an example.

The dynamic equation for the knee joint takes the form

$$
\tau_{\text {knee }}=M \ddot{\theta}_{\mathrm{k}}+G\left(\theta_{\mathrm{k}}\right)
$$

where $M$ is the moment of inertia (which is a constant) and $G\left(\theta_{\mathrm{k}}\right)$ is the gravity term.

Using (7)-(12), the control law for the knee joint can be expressed as

$$
\begin{aligned}
\tau_{\text {knee }}= & K_{\mathrm{p}}^{\prime}\left(0-\theta_{\mathrm{k}}\right)-K_{\mathrm{d}}^{\prime} \dot{\theta}_{\mathrm{k}}+K_{\mathrm{amp}} \cdot \max \left(x_{2}, 0\right) \cdot \tau_{\text {int. }} . \\
& +\widehat{G}\left(\theta_{\mathrm{k}}\right)
\end{aligned}
$$

where $\widehat{G}\left(\theta_{\mathrm{k}}\right)$ is the gravity compensation term. The term $K_{\mathrm{p}}^{\prime}\left(0-\theta_{\mathrm{k}}\right)-K_{\mathrm{d}}^{\prime} \dot{\theta}_{\mathrm{k}}$ represents high impedance control (i.e., position control), and $K_{\mathrm{amp}} \cdot \max \left(x_{2}, 0\right) \cdot \tau_{\text {int. }}$ represents low impedance control (i.e., force amplification control). In these expressions, $\tau_{\text {int. }}$ is the interaction torque generated during the cooperative walking process, $x_{1}$ and $x_{2}$ represent the extension and flexion neurons of the left hip joint, respectively, $K_{\mathrm{p}}^{\prime}=K_{\mathrm{p}} \cdot \max \left(x_{1}, 0\right), K_{\mathrm{p}}=20 \mathrm{Nm} /{ }^{\circ}\left({ }^{\circ}\right), K_{\mathrm{d}}^{\prime}=$ $K_{\mathrm{d}}=1.5 \mathrm{~N} \mathrm{~ms} /{ }^{\circ}\left({ }^{\circ}\right)$, and $K_{\mathrm{amp}}=2.5$. The alternate excitation mechanism of the extension and flexion neurons enables the knee joint to keep upright as the hip joint extends and the lower thigh to move forward naturally as the hip joint bends.

As the gravity compensation term can be rectified according to the measured joint torques under a set of static joint positions, we can assume that the gravity term has been fully compensated where we establish the closed-loop control 


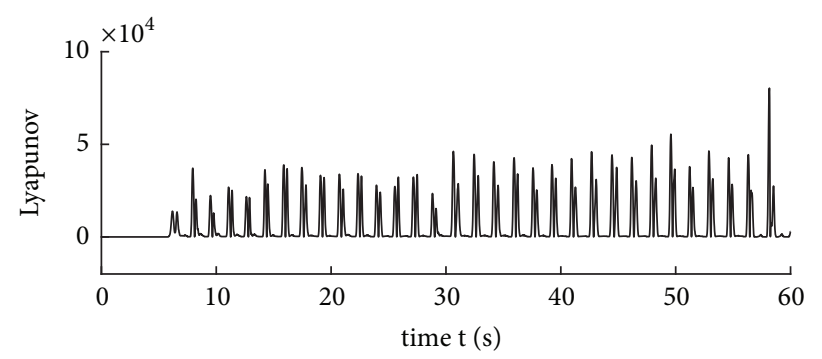

FIGURE 7: The variation of the Lyapunov function with time. The figure inside the graph is an enlarged partial view, which reveals more clearly that $V\left(\theta_{\mathrm{k}}, \dot{\theta}_{\mathrm{k}}\right) \geq 0$.

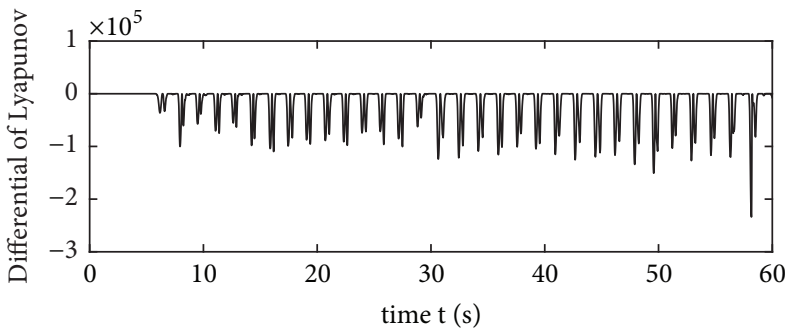

FIgURE 8: The variation of the Lyapunov time-derivative function with time. The figure inside the graph is an enlarged partial view, which reveals more clearly that $\dot{V}\left(\theta_{\mathrm{k}}, \dot{\theta}_{\mathrm{k}}\right) \leq 0$.

system (we therefore ignore them in what follows). The closed-loop control system can thus be established using (12) and (13) giving

$$
M \ddot{\theta}_{\mathrm{k}}+K_{\mathrm{d}}^{\prime} \dot{\theta}_{\mathrm{k}}+K_{\mathrm{p}}^{\prime} \theta_{\mathrm{k}}=K_{\mathrm{amp}} \cdot \max \left(x_{2}, 0\right) \cdot \tau_{\text {int. }} \cdot
$$

The Lyapunov function of the system can be written as

$$
V\left(\theta_{\mathrm{k}}, \dot{\theta}_{\mathrm{k}}\right)=\frac{1}{2} M \dot{\theta}_{\mathrm{k}}^{2}+\frac{1}{2} K_{\mathrm{p}}^{\prime}\left(0-\theta_{\mathrm{k}}\right)^{2}
$$

The time differential of the Lyapunov function is thus

$$
\dot{V}\left(\theta_{\mathrm{k}}, \dot{\theta}_{\mathrm{k}}\right)=\dot{\theta}_{\mathrm{k}} M \ddot{\theta}_{\mathrm{k}}+K_{\mathrm{p}}^{\prime} \theta_{\mathrm{k}} \dot{\theta}_{\mathrm{k}}
$$

According to (14), we have

$$
M \ddot{\theta}_{\mathrm{k}}=-K_{\mathrm{d}}^{\prime} \dot{\theta}_{\mathrm{k}}-K_{\mathrm{p}}^{\prime} \theta_{\mathrm{k}}+K_{\mathrm{amp}} \cdot \max \left(x_{2}, 0\right) \cdot \tau_{\text {int. }}
$$

Substituting (17) into (16) then gives an expression for the time differential of the Lyapunov function of the form

$$
\dot{V}\left(\theta_{\mathrm{k}}, \dot{\theta}_{\mathrm{k}}\right)=-K_{\mathrm{d}}^{\prime} \dot{\theta}_{\mathrm{k}}^{2}+K_{\mathrm{amp}} \cdot \max \left(x_{2}, 0\right) \cdot \tau_{\text {int. }} \cdot \dot{\theta}_{\mathrm{k}} \cdot
$$

According to Lyapunov stability theory, a system is asymptotically stable provided the following two conditions are met:

(i) The Lyapunov function is such that $V\left(\theta_{\mathrm{k}}, \dot{\theta}_{\mathrm{k}}\right) \geq 0$.

(ii) The time differential of the Lyapunov function is such that $\dot{V}\left(\theta_{\mathrm{k}}, \dot{\theta}_{\mathrm{k}}\right) \leq 0$.
Equation (15) implies that $V\left(\theta_{\mathrm{k}}, \dot{\theta}_{\mathrm{k}}\right) \geq 0$. However, as $\tau_{\text {int. }}$ and $\dot{\theta}_{\mathrm{k}}$ in (18) are parameters that vary over time, it is difficult to determine directly whether the condition $\dot{V}\left(\theta_{\mathrm{k}}, \dot{\theta}_{\mathrm{k}}\right) \leq 0$ is obeyed. In this paper, real-time data is obtained for $\tau_{\text {int. }}$ and $\dot{\theta}_{\mathrm{k}}$ from walking experiments and $\dot{V}\left(\theta_{\mathrm{k}}, \dot{\theta}_{\mathrm{k}}\right)$ values are then calculated using (18). The details of how the walking experiments were implemented are given in Section 5.

Figures 7 and 8 present graphs showing how the Lyapunov function and its time differential were found to vary as a function of time. The results reveal that the conditions $V\left(\theta_{\mathrm{k}}, \dot{\theta}_{\mathrm{k}}\right) \geq 0$ and $\dot{V}\left(\theta_{\mathrm{k}}, \dot{\theta}_{\mathrm{k}}\right) \leq 0$ are both met. Thus, the results satisfy the Lyapunov stability criteria and so the control system is confirmed to be stable.

Figure 9 further illustrates the stability of the system. Figure 9(a) shows that the system is stable near the equilibrium point and that the system has a domain that is attractive. Thus, the asymptotic stability of the system is proved. Meanwhile, kinematic limit cycles are shown in Figure 9(b) which also demonstrate the stability of the knee joint motion.

2.5. Simulations. We first carried out computer simulations to verify the rationality of using the proposed control system as a method of generating hip-knee joint movement while walking.

The simulations were based on a simplified unilateral leg model consisting of two joints and two links, as shown in Figure 10. We assume that the hip joint is connected to the base and the terminal end of the leg is free and has no contact with the environment. The parameters used to model the two links are given in Table 2.

The dynamic equation was established using Lagrange equations and the simulation method used is illustrated in 


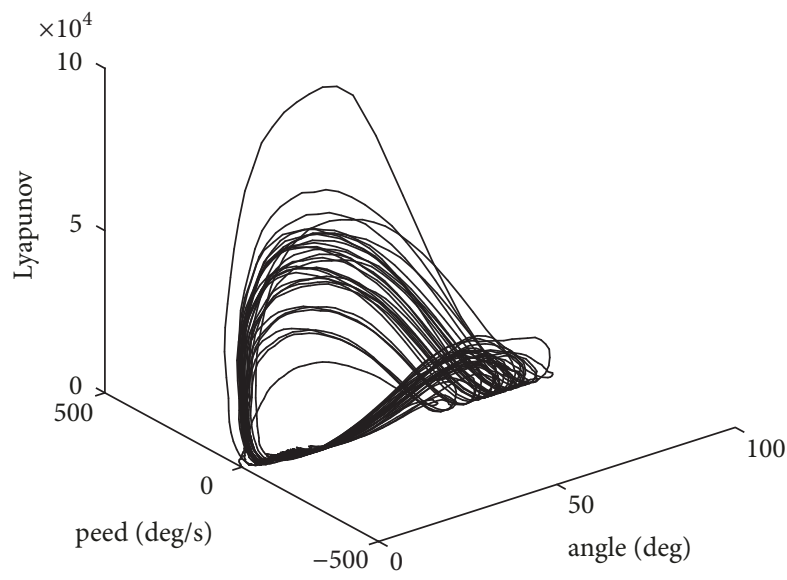

(a) Equilibrium point

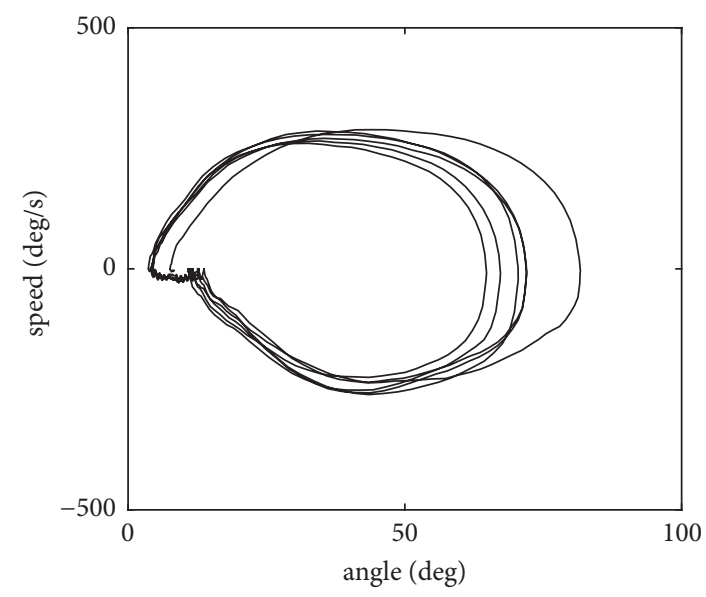

(b) Limit cycle

FIGURE 9: Stable equilibrium point and limit cycles corresponding to the motion of the knee joint.

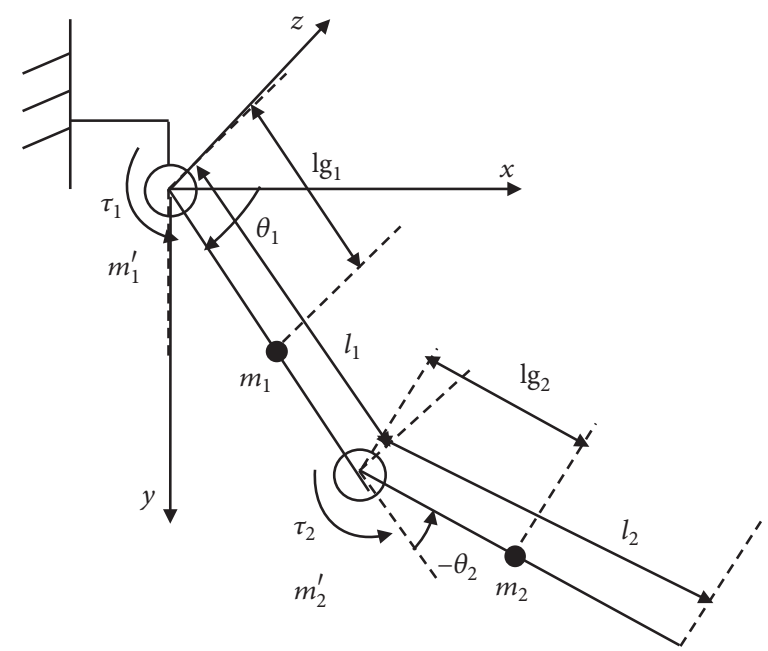

FIGURE 10: Simplified model of a leg.

TABLE 2: The parameters of the links used in the model.

\begin{tabular}{lccc}
\hline & \multicolumn{3}{c}{ Parameter } \\
& Mass $(\mathrm{kg})$ & Length $(\mathrm{m})$ & Center of mass $(\mathrm{m})$ \\
\hline First link & 1.0 & 0.5 & 0.25 \\
Second link & 1.0 & 0.5 & 0.25 \\
\hline
\end{tabular}

Figure 11. The hip joint and knee joint torques were calculated using (5) and (10), respectively.

When the gain $C$ of the CPGs is zero (i.e., there is no feedback to the CPGs), the trajectories of the joints during one extension/flexion period are as shown in Figure 12. These kinds of trajectories correspond to "master" motion, and the frequency is about $1.0 \mathrm{~Hz}$. The upper part of Figure 12 shows the desired and real hip joint trajectories, and the lower part shows the knee joint trajectory. The trajectories show that in the stance phase the hip joint makes an extension motion, and the knee joint keeps straight. In the swing phase, along with hip joint flexion, the knee joint first flexes and then extends.
This result coincides with the design requirements mentioned in Section 2.5. To be more explicit and reveal the motion more clearly, the movement of the "leg" in the sagittal plane is illustrated in Figure 13.

As human-robot interaction is not considered in the current simulation, we change the value of the gain $C$ to 0.5 in order to evaluate the flexibility of this proposal and generate master/slave motion. Thus, we define the input signal to the CPGs to be $\sin (\pi t) \cdot C$.

Figure 14 shows the joint trajectories obtained during one extension/flexion period when $C=0.5$. These trajectories correspond to a kind of slave motion. The frequency found corresponds to $0.5 \mathrm{~Hz}$, which coincides with that of the input. The upper part again shows the desired and real hip joint trajectories, while the lower part shows the knee joint trajectory. It can be seen that in the stance phase the hip joint undergoes extension and the knee joint keeps straight. In the swing phase, along with flexion of the hip joint, the knee joint first flexes and then extends. The motion of the leg in the sagittal plane is shown in Figure 15. 


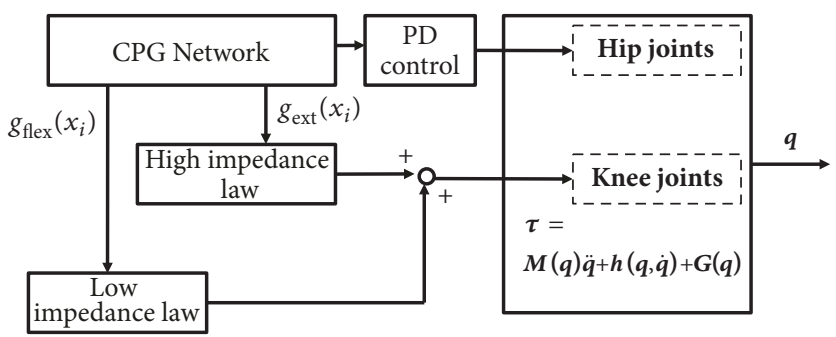

FIgURE 11: The simulation method used.

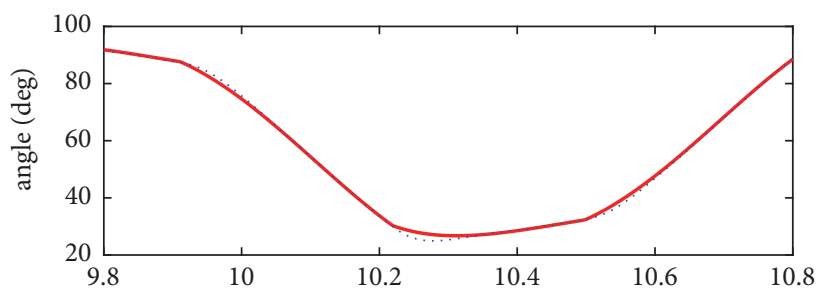

— Desired hip joint angle Real hip joint angle

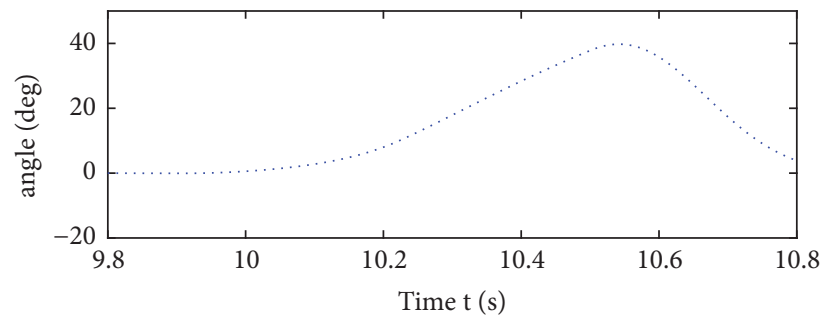

FIGURE 12: The joint trajectories when $C=0$.

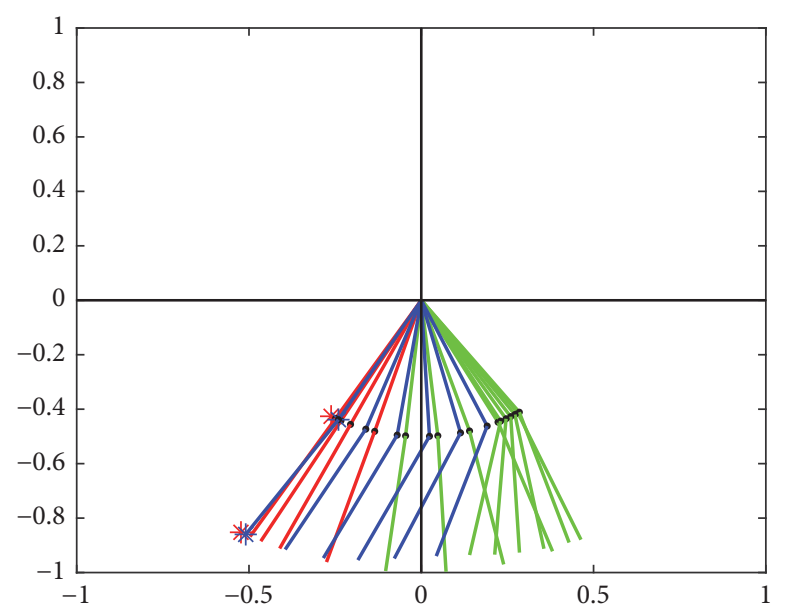

Figure 13: The movement of the joints in the sagittal plane $(C=0)$.

The simulations above preliminarily demonstrate that the proposed HRI-based control method (consisting of hip joint CPG control, knee joint hierarchical impedance control, and hip-knee joint linkage control) is able to effectively generate natural hip-knee joint movement.

\section{Experiments}

3.1. Participants and Experimental Setup. One healthy participant took part in our experiments (a 35-year-old male, $1.70 \mathrm{~m}$ tall, weighing $65 \mathrm{~kg}$ ). The experiments were conducted 

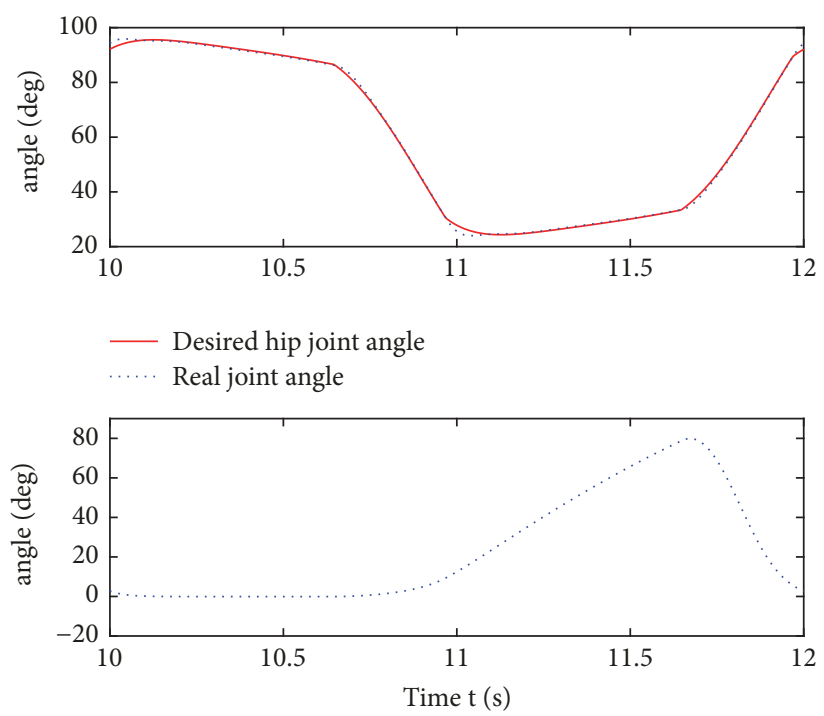

FIgURE 14: The joint trajectories when $C=0.5$.

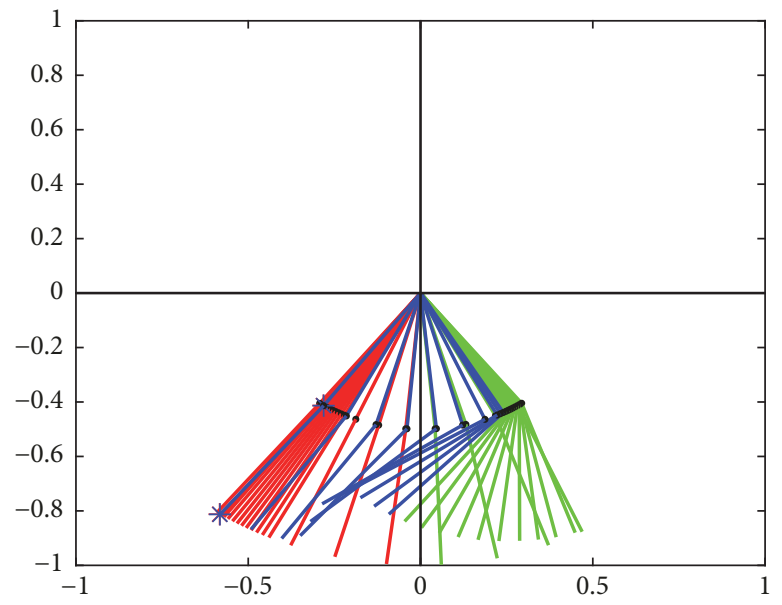

FIGURE 15: The movement of the joints in the sagittal plane $(C=0.5)$.

in agreement with the local institution's ethics regulations, and the participant signed a written consent form.

To test our approach, we used a wearable walking assist robot called Curara ${ }^{\circledR}$ to assist the hip joint flexion and extension and knee joint motion of our subject in the sagittal plane (vertical plane through vertical axis of the body and all of parallel plane) during walking. As shown in Figure 16, the Curara system consists of a lumbar fixing device, knee and hip joint bases, two knee joint fixing devices, thigh and shank links on the left and right sides, harmonic drive gears, builtin joint torque sensors, and an external computer. Each of the actuators has a built-in torque sensor consisting of three strain gauges fixed onto the flex-spline of the harmonic drive gear. If there is any difference between the user's and suit's movements, then the mutual joint torque is measured. The sensitivity of the torque sensor is about $7.2 \mathrm{Nm} / \mathrm{V}$. The type and main parameters of the actuators are shown in Table 3.

Powerful Velcro is used to adhere the base of the hip joint to the lumbar fixing device. The lumbar region fixing device is made of a nylon composite material which gives a good fit to the human body. It also allows a certain adduction-abduction-driven degree of freedom and a certain axial degree of freedom in the hip joint. This kind of flexible design enables direction changes to be accomplished more freely during walking. To achieve a lightweight design, the knee joint fixing device, bases of the hip and knee joints, and connecting thigh and shank rods are made of nanoresin material. As a result, the total weight of the whole device is about $5.3 \mathrm{~kg}$. The Ubuntu platform on an external computer (PCM-3363) was used to develop the software needed for the current experiments.

3.2. Experimental Protocol. The participant, wearing the Curara device on both legs, was asked to walk as he would normally. A photograph of the participant walking is shown in Figure 17. The Curara device was fastened via the attachment cuffs to the middles of the thighs and tops of the calves of the participant. The pelvic module was further 
TABLE 3: Type and main parameters of the actuators.

\begin{tabular}{lccc}
\hline & Type & Parameter & Value \\
\hline Retarder & SHF11-50 & Maximum output torque (Nm) & 17 \\
& & Retarder ratio & 50 \\
Motor & X-67087 & Maximum speed (r/min) & 3000 \\
& & Maximum current (A) & 3.2 \\
\hline
\end{tabular}
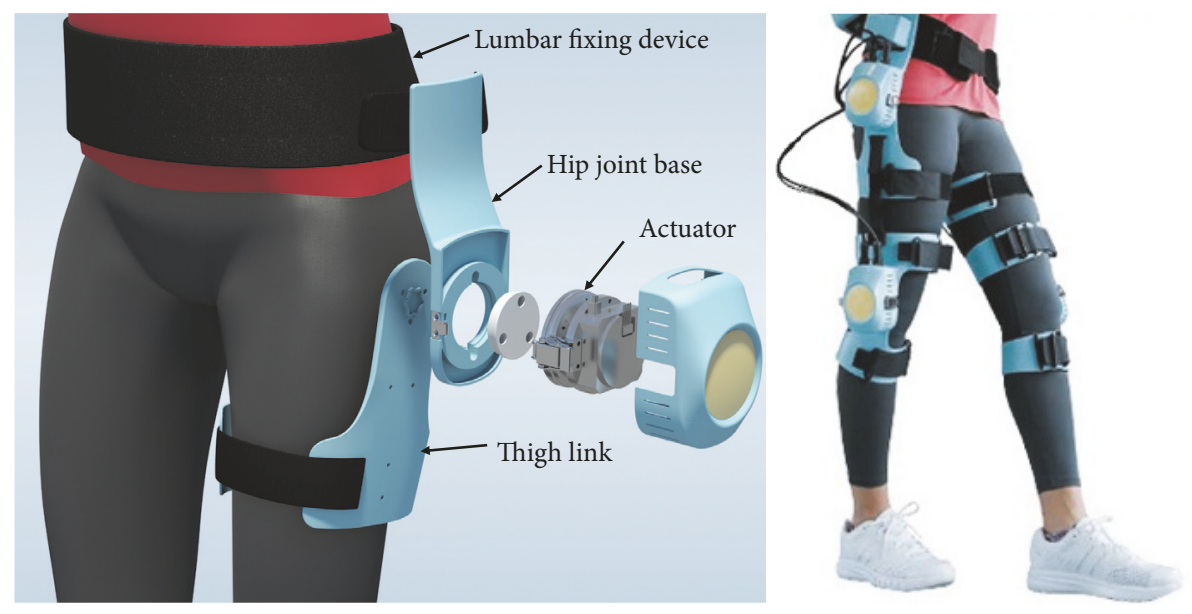

FIGURE 16: The wearable walking assist suit Curara.
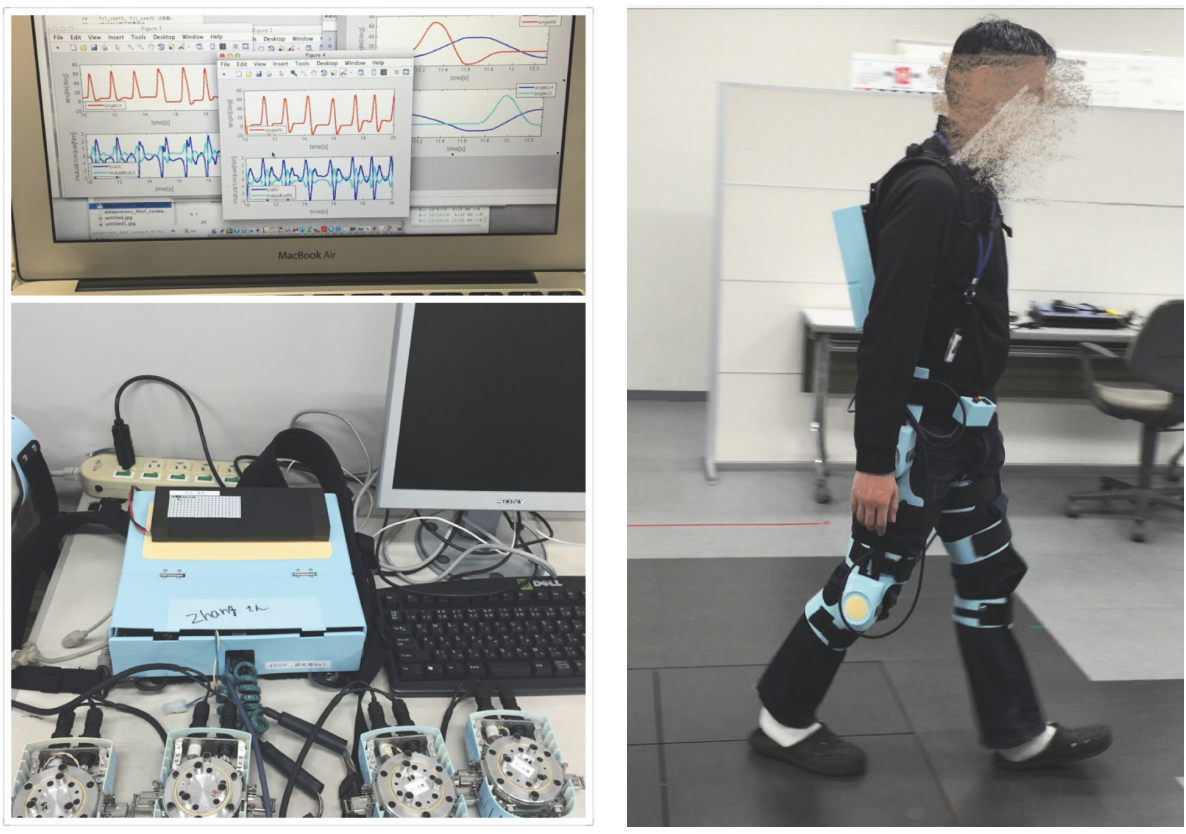

FIGURE 17: The experimental scenario.

attached to the participant's waist using a belt. The tests were carried out at ground level in our laboratory and involved the participant walking to and fro in a straight line of length $5 \mathrm{~m}$. Each walking test lasted $1 \mathrm{~min}$. After this period, the controller was stopped, and the user took off the wearable robot.
The torques and angular positions of the robot's joints were recorded using the sensors within the Curara device. Resistive foot-pressure sensors were placed on the left and right insoles of the participant and used as switches to detect heel-strike and toe-off events. All data (recorded and derived) was separated into "strides", i.e., intervals between 


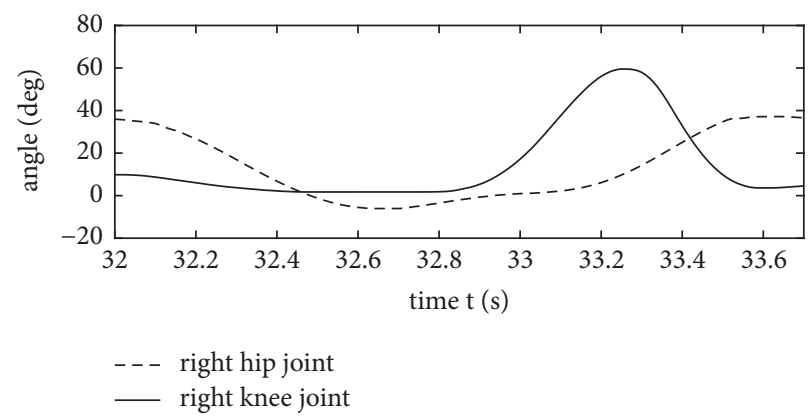

FIGURE 18: Typical trajectories of the right hip and knee joints. The periods labeled relate to different stages in the participant's gait corresponding to double support (DS), swing, and single-support stance.

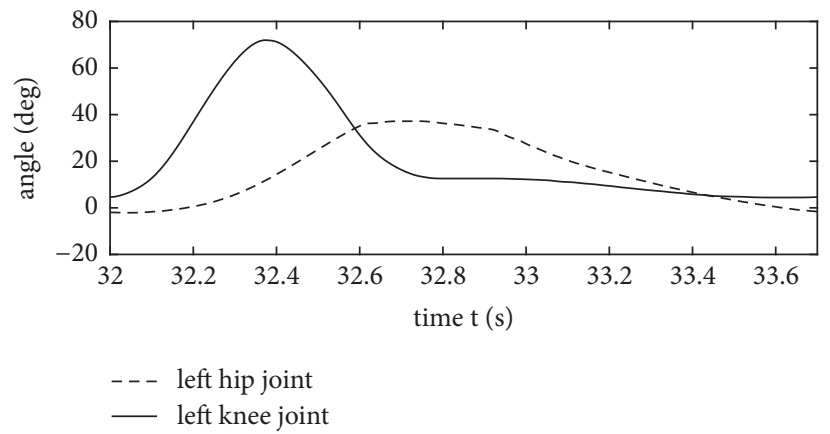

FIGURE 19: Typical trajectories of the left hip and knee joints. The periods labeled relate to different stages in the participant's gait corresponding to double support (DS), swing, and single-support stance.

two consecutive left heel-strikes, using the pressure data from the insole sensors. In each stride, we computed the participant's gait (left and right heel-strike and toe-off) as an indicator of the symmetry of the step.

3.3. Experimental Results. The trajectories of the right and left hip-knee joints recorded during walking are shown in Figures 18 and 19, respectively. The dotted and solid lines represent the trajectory of the hip and knee joints in a gait cycle, which is for one typical stride. The results demonstrate that the knee joint keeps upright as the hip joint extends, and the knee joint bends at first and then extends as the hip joint bends. These results coincide with the expectations of the design.

If we compare the experimental results with the simulations, the kinematic profiles can be seen to be very similar. In both kinematic profiles (demonstrated in Figures 14 and 18), along with the hip joints undergoing flexion and extension, the knee joints keep straight to maintain posture in the stance phase and undergo flexion-extension in the swing phase. However, as the effect of the human-robot-environment interaction was omitted in the simulations, the amplitudes of the joint angles are greater therein. In contrast, the human and robot were combined as one system in the walking experiments, and so these kinematic profiles correspond to cooperative walking. The assistive robot adapts to the human movement, and so the amplitudes of the joint angles are smaller.

Figures 20 and 21 show how the right and left knee joint torque changed over time (gait) during one complete walking cycle, respectively. It can be seen that the changes in the knee joint torque are mild within $0.1 \mathrm{~s}$ of the time at which the support and swing stages switch (the $50 \%$ gate point). Furthermore, and the differences between the maximum and minimum torques at the right and left knee joints are 1.35 and $1.55 \mathrm{Nm}$, respectively. These results indicate that the knee joint torque changes relatively smoothly as the system switches between high and low impedance control. From these results it can be concluded that the proposed control method is an effective and feasible way of generating natural hip-knee kinematics.

\section{Discussion}

In this article, we present simulations and experiments that were recently conducted to evaluate a new hybrid assistive approach to generating natural angular movement of hip and knee joints during walking. The approach combines a CPG network with hierarchical impedance control. The work presented provides evidence that (i) the hybrid approach is stable, and (ii) the CPG network allows natural movement patterns to be realized.

The requirement to provide assistance depending on the behavior of the user is of prime interest in the framework of assist-as-needed assistive robotics. We believe that the CPG network-based framework presented in this article nicely complements other approaches based on compliance [28-31], adaptation [28, 32, 33], or adaptive learning of a dynamical model for the task at hand [34]. In particular, our approach relies on the concept of motor primitives, which first emerged 


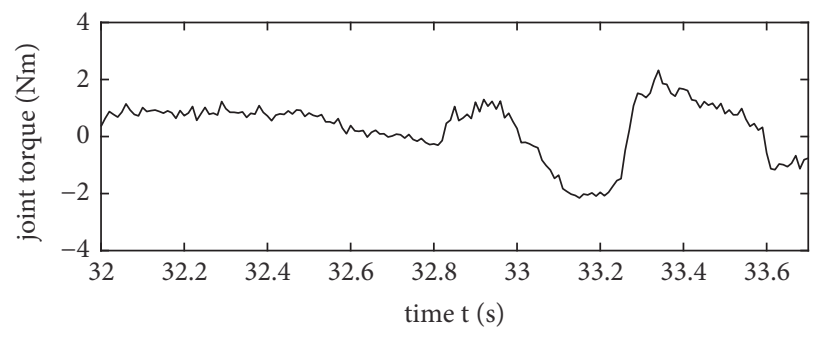

Figure 20: The torque recorded at the right knee joint. The periods labeled relate to different stages in the participant's gait corresponding to double support (DS), swing, and single-support stance.

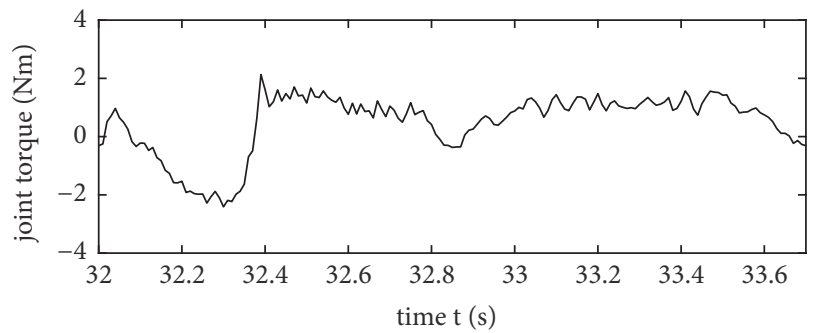

FIGURE 21: The torque recorded at the left knee joint. The periods labeled relate to different stages in the participant's gait corresponding to double support (DS), swing, and single-support stance.

in biology $[35,36]$ and is now extensively used in robotics [37]. Assistive and rehabilitation robotics fall within the overlap between both fields and should therefore be an ideal testbed for the concept.

One of the main characteristics of our approach is its capacity to robustly synchronize the frequency of the output signal with the input signal. Furthermore, the level of synchronization can be adjusted using the input weight $C$ to obtain a mixture of master/slave motion. Synchronization is a ubiquitous phenomenon in biology [38]. During locomotion, it is observed at the spinal CPG level to guarantee coordination of the locomotion pattern [39]. Here, we have attempted to implement the synchronization mechanisms required by means of CPGs that use the Matsuoka mathematical model [27] to generate oscillatory behavior. The profiles presented in Figure 2 (Section 2.2) show that the CPG constructed can easily transform from stable autonomous oscillation to stable synchronous oscillation. The results thus show that the proposed method is capable of exhibiting master-slave motion.

The innovative aspect of our approach is that for the first time in the field of assistive robotics an attempt has been made to combine biologically inspired motion control with traditional impedance control. Together with the rhythmic control of the hip joint (achieved using the neural rhythm generator), control is also needed over the mechanical impedance to generate appropriate torques at the knee joints in order to maintain posture during walking. The stiffness and viscosity of the knee joints have to be increased during the stance phase to avoid collapse. They are then decreased during the swing phase to allow the wearer to step forward uninhibited. The high and low impedance control laws to maintain the corresponding posture are switched between depending on the state of the hip joint neuron on the ipsilateral side.
Neurophysiological studies on quadruped animals suggest that parallel pathways are involved in the control of rhythmic movement and posture [40].

In the coming years, the biggest challenge for us will be to transfer these concepts to providing actual walking assistance, i.e., to studies with genuine patients. However, we believe that our approach opens up some promising avenues. There are two reasons for this: (i) no sensors are placed on the human body (all sensors are contained within the device), and (ii) the master-slave motion assistance it provides is very flexible (therefore, various assist-as-needed protocols can be explored by, for example, adjusting the input weight gain to achieve the master-slave motion assistance required).

\section{Conclusions}

In this article, biological control theory is introduced to improve human-robot interaction flexibility and multijoint autonomy in walking assist devices. A novel HRI-based control method that includes hip joint CPG control, knee joint hierarchical impedance control, and hip-knee joint linkage control is explored.

CPG networks allow adequate human-robot interaction to be achieved and improve the flexibility with respect to master/slave motion. Mutual inner-inhibition is useful for maintaining an antiphase relationship between the left and right hip joints (necessary for stable walking assistance in the complicated HRI environment). An impedance structured control was designed to control knee joint movement. A hipknee joint linkage mechanism was also designed to achieve natural hip-knee joint motion.

The most significant contribution of the proposed control framework is that it opens up a new way of creating new 
assistive methods for robotic suits that avoid complicated kinematic and dynamic modeling. At the same time, it improves the flexibility and autonomy of multijoint systems. Simulations and walking experiments using a wearable device were used to prove the effectiveness of the proposed control method to generate natural hip-knee joint motion during walking. Finally, the stability of the hybrid control method was studied using Lyapunov stability theory. The experimental results show that the Lyapunov function is positive definite and the time differential of the Lyapunov function is negative definite (thus satisfying the Lyapunov stability criteria). In addition, the system is stable near the equilibrium point and has an attractive domain. Limit cycles for the knee motion were also generated that prove the control system is stable.

In future work, the proposed control framework will be used in conjunction with a wearable robotic suit (provided by the Hashi Laboratory of the Shinshu University in Japan) to perform walking assist experiments involving real patients.

\section{Data Availability}

The data used to support the findings of this study are available from the corresponding author upon request.

\section{Conflicts of Interest}

The authors declare that they have no conflicts of interest.

\section{Acknowledgments}

This work was supported by the National Natural Science Foundation of China (51505048), Natural Science Foundation Project of CQ CSTC (cstc2016jcyjA0416), and a Science and Technology Project of the Chongqing Municipal Education Commission (KJ1500526). The authors thank the Hashi Laboratory of the Shinshu University in Japan for its technical and financial support for this research.

\section{References}

[1] G. Colombo, M. Joerg, R. Schreier, and V. Diets, "Treadmill training of paraplegic patients using a robotic orthosis," J. Rehabil. Res. Dev, vol. 37, pp. 693-700, 2000.

[2] J. F. Veneman, R. Kruidhof, E. E. G. Hekman, R. Ekkelenkamp, E. H. F. Van Asseldonk, and H. Van Der Kooij, "Design and evaluation of the LOPES exoskeleton robot for interactive gait rehabilitation," IEEE Transactions on Neural Systems and Rehabilitation Engineering, vol. 15, no. 1, pp. 379-386, 2007.

[3] D. Sanz-Merodio, M. Cestari, J. C. Arevalo, X. A. Carrillo, and E. Garcia, "Generation and control of adaptive gaits in lower-limb exoskeletons for motion assistance," Advanced Robotics, vol. 28, no. 5, pp. 329-338, 2014.

[4] A. Esquenazi, M. Talaty, A. Packel, and M. Saulino, "The ReWalk powered exoskeleton to restore ambulatory function to individuals with thoracic-level motor-complete spinal cord injury," American Journal of Physical Medicine \& Rehabilitation, vol. 91, no. 11, pp. 911-921, 2012.
[5] K. A. Strausser and H. Kazerooni, "The development and testing of a human machine interface for a mobile medical exoskeleton," in Proceedings of the 2011 IEEE/RSJ International Conference on Intelligent Robots and Systems: Celebrating 50 Years of Robotics, IROS'11, pp. 4911-4916, September 2011.

[6] H. Kazerooni and R. Steger, "The Berkeley Lower Extremity Exoskeleton," Journal of Dynamic Systems, Measurement, and Control, vol. 128, no. 1, p. 14, 2006.

[7] E. Guizzo and H. Goldstein, "The rise of the body bots," IEEE Spectrum, vol. 42, no. 10, pp. 50-56, 2005.

[8] C. J. Walsh, K. Endo, and H. Herr, "A quasi-passive leg exoskeleton for load-carrying augmentation," International Journal of Humanoid Robotics, vol. 4, no. 3, pp. 487-506, 2007.

[9] Y. Sankai, "HAL: Hybrid assistive limb based on cybernics," Springer Tracts in Advanced Robotics, vol. 66, pp. 25-34, 2010.

[10] E. Commission, "Population and Housing census," 2018, http://ec.europa.eu/eurostat/web/population-and-housing-census/statistics-illustrated.

[11] "A. Administration for Community Living, Aging and Disability in America," 2018, https://www.acl.gov/aging-and-disability-in-america.

[12] H. Kazerooni, "The Berkeley lower extremity exoskeleton (BLEEX)," Field and Service Robotics, vol. 25, no. 1, pp. 9-15, 2006.

[13] Z. Yang, Y. Zhu, X. Yang, and Y. Zhang, "Impedance Control of Exoskeleton Suit Based on Adaptive RBF Neural Network," in Proceedings of the 2009 International Conference on Intelligent Human-Machine Systems and Cybernetics, pp. 122-127, Hangzhou, Zhejiang, China, 2012.

[14] D. Sanz-Merodio, M. Cestari, J. C. Arevalo, and E. Garcia, "Control Motion Approach of a Lower Limb Orthosis to Reduce Energy Consumption," International Journal of Advanced Robotic Systems, vol. 9, no. 6, p. 232, 2012.

[15] L. Wang, S. Wang, E. H. F. Van Asseldonk, and H. Van Der Kooij, "Actively controlled lateral gait assistance in a lower limb exoskeleton," in Proceedings of the 2013 26th IEEE/RSJ International Conference on Intelligent Robots and Systems: New Horizon, IROS 2013, pp. 965-970, Japan, November 2013.

[16] H. Kawamoto, S. Taal, H. Niniss et al., "Voluntary motion support control of Robot Suit HAL triggered by bioelectrical signal for hemiplegia," in Proceedings of the 32nd Annual International Conference of the IEEE Engineering in Medicine and Biology Society (EMBC '10), pp. 462-466, Buenos Aires, Argentina, September 2010.

[17] G. P. Rosati Papini and C. A. Avizzano, "Transparent force control for Body Extender," in Proceedings of the 2012 21st IEEE International Symposium on Robot and Human Interactive Communication, RO-MAN 2012, pp. 138-143, France, September 2012.

[18] L. Righetti, J. Buchli, and A. J. Ijspeert, "Dynamic Hebbian learning in adaptive frequency oscillators," Physica D: Nonlinear Phenomena, vol. 216, no. 2, pp. 269-281, 2006.

[19] N. L. Tagliamonte, F. Sergi, G. Carpino, D. Accoto, and E. Guglielmelli, "Human-robot interaction tests on a novel robot for gait assistance," in Proceedings of the 2013 IEEE 13th International Conference on Rehabilitation Robotics, ICORR 2013, USA, June 2013.

[20] X. Zhang and M. Hashimoto, "Synchronization-based trajectory generation method for a robotic suit using neural oscillators for hip joint support in walking," Mechatronics, vol. 22, no. 1, pp. 33-44, 2011. 
[21] S. Lee and Y. Sankai, "Minimizing the physical stress by virtual impedance of exoskeleton robot in swinging motion with power assist system for lower limb," Journal of the Japan Society of Mechanical Engineers, vol. 71, no. 705, pp. 1686-1695, 2005.

[22] H. Kazerooni, The Berkeley lower extremity exoskeleton (BLEEX, Field and Service Robotics, vol. 25, Field and Service Robotics, 1 edition, 2006.

[23] S. Oh, E. Baek, S.-K. Song, S. Mohammed, D. Jeon, and K. Kong, "A generalized control framework of assistive controllers and its application to lower limb exoskeletons," Robotics and Autonomous Systems, vol. 73, pp. 68-77, 2015.

[24] V. Rajasekaran, J. Aranda, A. Casals, and J. L. Pons, "An adaptive control strategy for postural stability using a wearable robot," Robotics and Autonomous Systems, vol. 73, pp. 16-23, 2015.

[25] X. Zhang and M. Hashimoto, "Inhibitory connections between neural oscillators for a robotic suit," in Proceedings of the 2011 IEEE International Conference on Robotics and Automation, ICRA 2011, pp. 4182-4187, China, May 2011.

[26] X. Zhang, T. Luo, and M. Hashimoto, "A human-robot interaction based coordination control framework for walking assist," in Proceedings of the 2016 IEEE International Conference on Robotics and Biomimetics, ROBIO 2016, pp. 1467-1472, China, December 2016.

[27] K. Matsuoka, "Mechanisms of frequency and pattern control in the neural rhythm generators," Biological Cybernetics, vol. 56, no. 5-6, pp. 345-353, 1987.

[28] A. Duschau-Wicke, J. Von Zitzewitz, A. Caprez, L. Lünenburger, and R. Riener, "Path control: A method for patient-cooperative robot-aided gait rehabilitation," IEEE Transactions on Neural Systems and Rehabilitation Engineering, vol. 18, no. 1, pp. 38-48, 2010.

[29] R. Riener, L. Lünenburger, S. Jezernik, M. Anderschitz, G. Colombo, and V. Dietz, "Patient-cooperative strategies for robot-aided treadmill training: First experimental results," IEEE Transactions on Neural Systems and Rehabilitation Engineering, vol. 13, no. 3, pp. 380-394, 2005.

[30] H. Vallery, E. H. F. Van Asseldonk, M. Buss, and H. Van Der Kooij, "Reference trajectory generation for rehabilitation robots: Complementary limb motion estimation," IEEE Transactions on Neural Systems and Rehabilitation Engineering, vol. 17, no. 1, pp. 23-30, 2009.

[31] E. H. F. Van Asseldonk, R. Ekkelenkamp, J. F. Veneman, F. C. T. Van Der Helm, and H. Van Der Kooij, "Selective control of a subtask of walking in a robotic gait trainer(LOPES)," in Proceedings of the 2007 IEEE 10th International Conference on Rehabilitation Robotics, ICORR'07, pp. 841-848, Netherlands, June 2007.

[32] S. K. Banala, S. H. Kim, S. K. Agrawal, and J. P. Scholz, "Robot assisted gait training with active leg exoskeleton (ALEX)," IEEE Transactions on Neural Systems and Rehabilitation Engineering, vol. 17, no. 1, pp. 2-8, 2009.

[33] S. Jezernik, G. Colombo, and M. Morari, "Automatic gaitpattern adaptation algorithms for rehabilitation with a 4-DOF robotic orthosis," IEEE Transactions on Robotics and Automation, vol. 20, no. 3, pp. 574-582, 2004.

[34] E. T. Wolbrecht, V. Chan, D. J. Reinkensmeyer, and J. E. Bobrow, "Optimizing compliant, model-based robotic assistance to promote neurorehabilitation," IEEE Transactions on Neural Systems and Rehabilitation Engineering, vol. 16, no. 3, pp. 286-297, 2008.

[35] E. Bizzi, V. C. K. Cheung, A. d'Avella, P. Saltiel, and M. Tresch, "Combining modules for movement," Brain Research Reviews, vol. 57, no. 1, pp. 125-133, 2008.
[36] C. B. Hart and S. F. Giszter, "A neural basis for motor primitives in the spinal cord," The Journal of Neuroscience, vol. 30, no. 4, pp. 1322-1326, 2010.

[37] S. Degallier and A. Ijspeert, "Modeling discrete and rhythmic movements through motor primitives: A review," Biological Cybernetics, vol. 103, no. 4, pp. 319-338, 2010.

[38] S. H. Strogatz, SYNC: The Emerging Science of Spontaneous Order, Hyperion Press, New York, NY, USA, 2003.

[39] A. J. Ijspeert, "Central pattern generators for locomotion control in animals and robots: a review," Neural Networks, vol. 21, no. 4, pp. 642-653, 2008.

[40] S. Mori, "Integration of posture and locomotion in acute decerebrate cats and in awake, freely moving cats," Progress in Neurobiology, vol. 28, no. 2, pp. 161-195, 1987. 


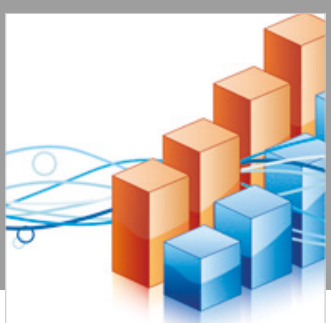

Advances in

Operations Research

\section{-n-m}
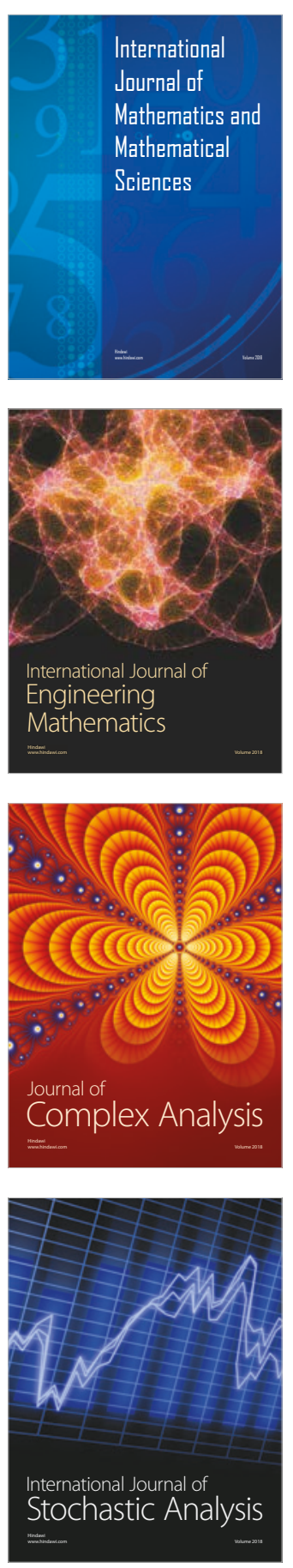
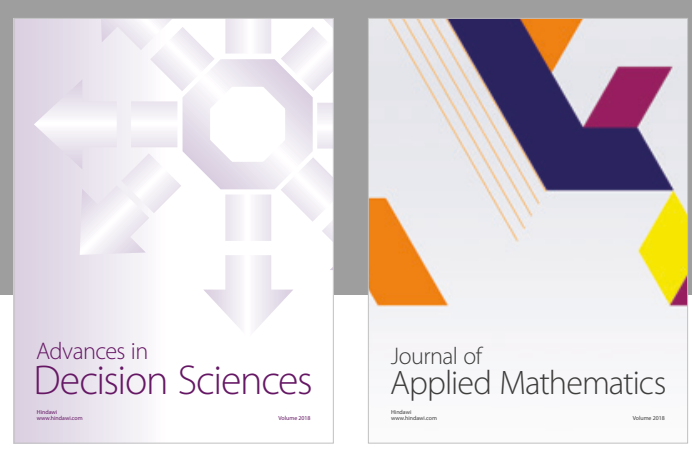

Journal of

Applied Mathematics
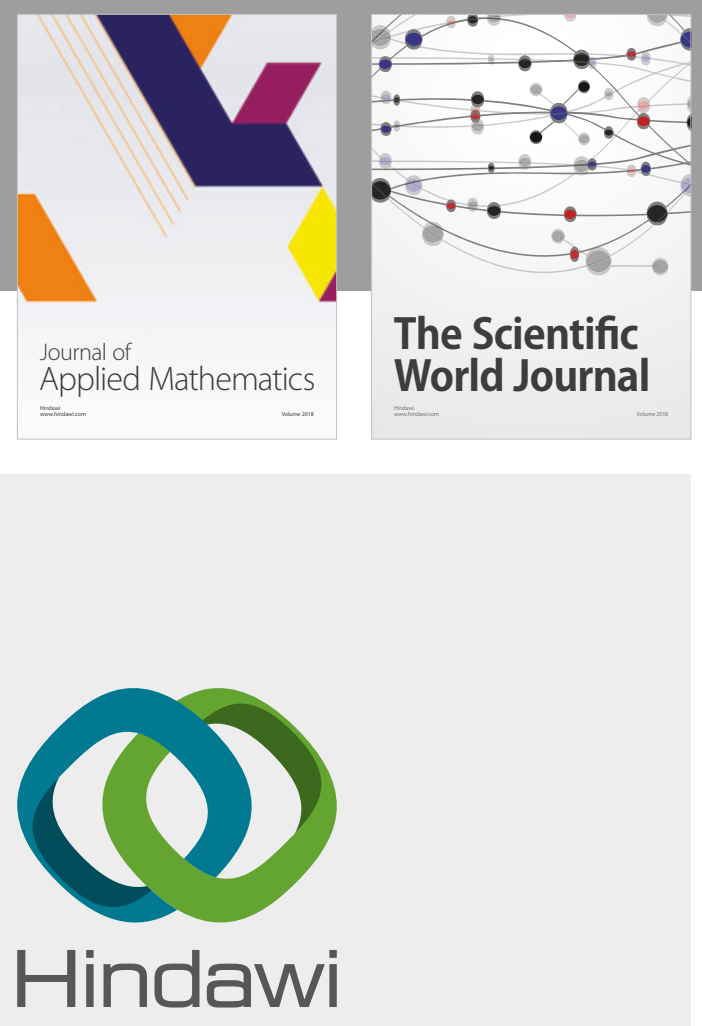

Submit your manuscripts at

www.hindawi.com

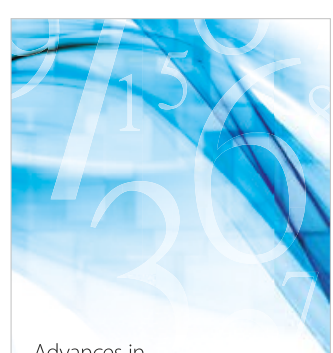

Advances in
Numerical Analysis
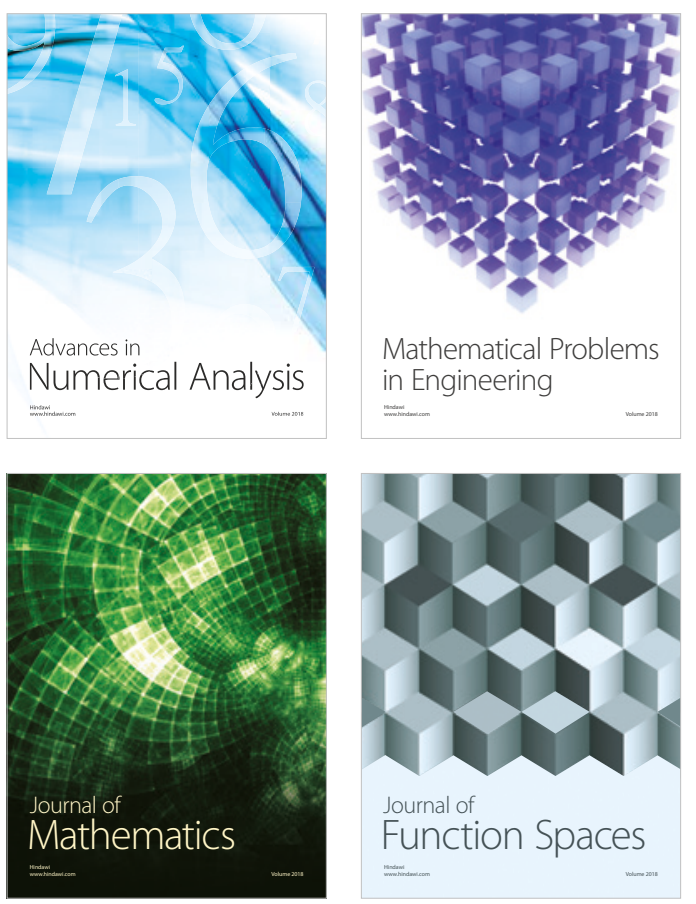

Mathematical Problems in Engineering

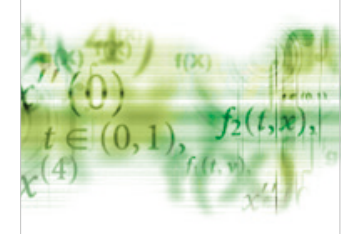

International Journal of

Differential Equations

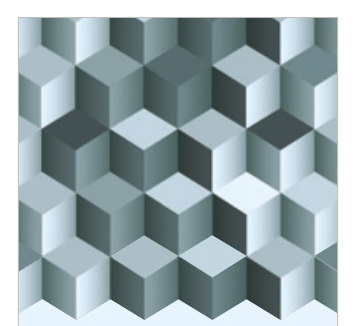

Journal of

Function Spaces

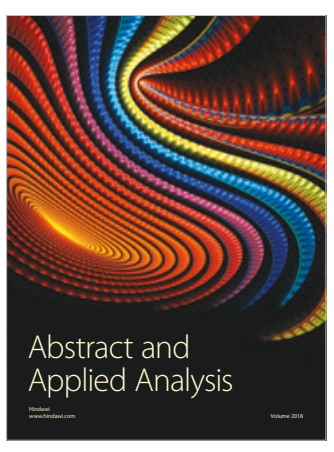

The Scientific

World Journal

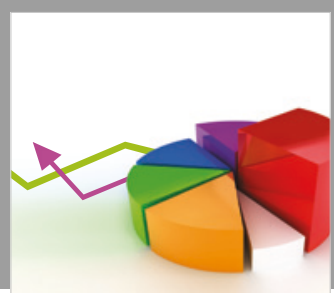

Journal of

Probability and Statistics
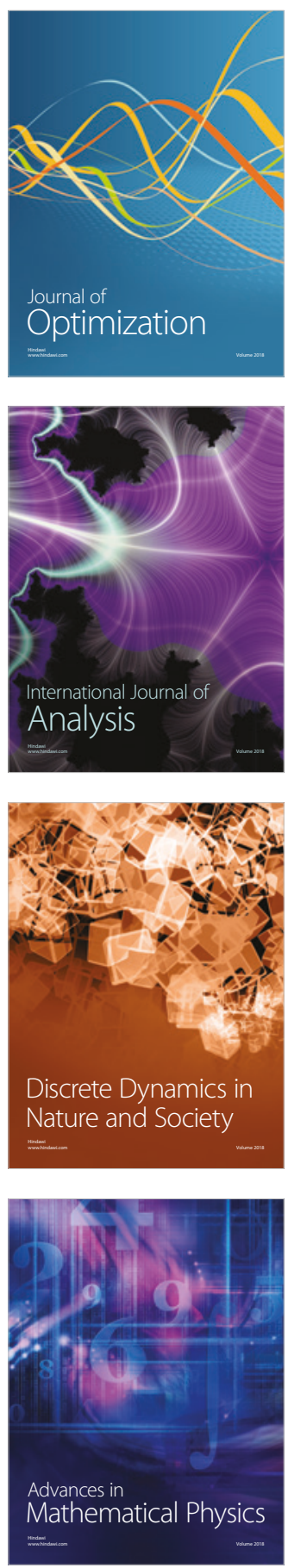